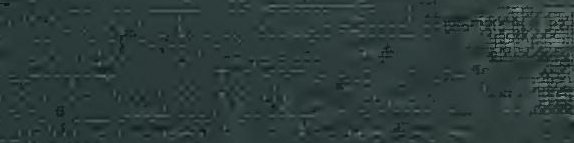

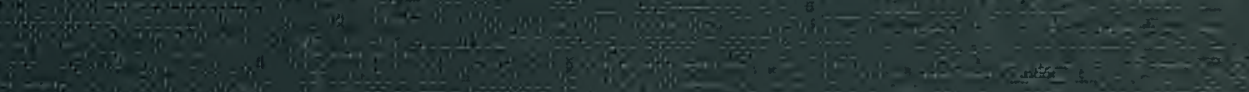

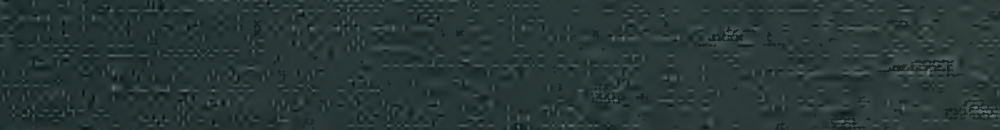

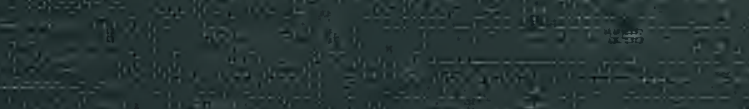

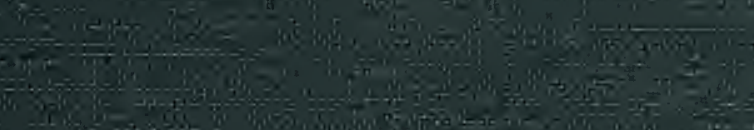

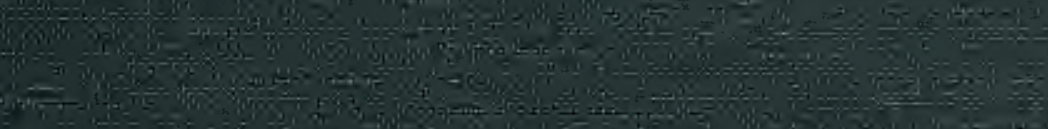
$=$

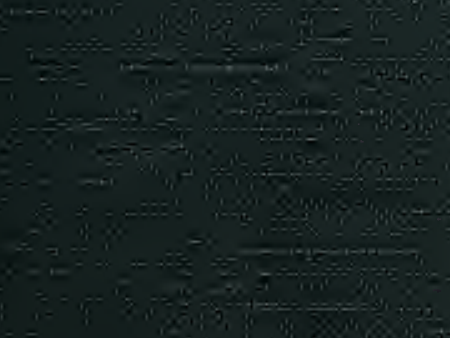

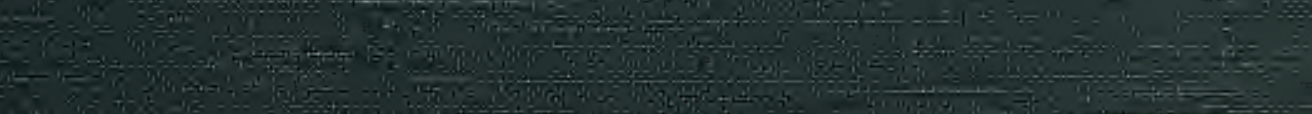

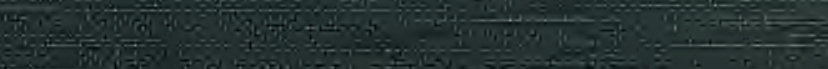$$
\text { (1) }
$$ 


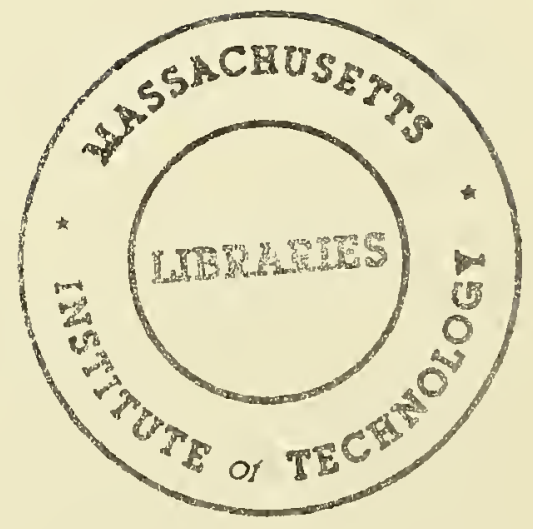




\section{Digitized by the Internet Archive in 2011 with funding from Boston Library Consortium Member Libraries}





\section{working poper} department of economics

REPUTATION IN THE SIMULTANEOUS PLAY

OF MULTIPLE OPPONENTS

\section{Drew Fudenberg} David M. Kreps

\section{massachusetts instifute of technology}

50 memorial drive cambridge, mass.02139 

REPUTATION IN THE SIMULTANEOUS PLAY

OF MULTIPLE OPPONENTS

Drew Fudenberg

David M. Kreps

Number 466

March 1987 


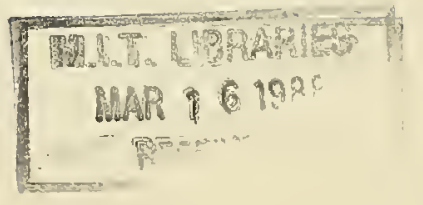




\title{
Reputation in the Simultaneous Play of Multiple Opponents *
}

Drew Fudenberg and David M. Kreps

March 1987

\begin{abstract}
Imagine that one player, the "incumbent," competes with several "entrants." Each entrant competes only with the incumbent, but observes play in all contests. Previous work shows that, as more and more entrants are added, the incumbent's reputation may dominate play of the game, if the entrants are faced in sequence. We identify conditions under which similar results obtain when the entrants are faced simultaneously, and we find specifications in which adding more simultaneous entrants has a dramatically different effect. We also show that, with either sequential or simultaneous play, incumbents need not prefer the situation in which their reputations can and do dominate play to the "informationally isolated" case in which each entrant observes only play in its own contest.
\end{abstract}

\section{Introduction}

This paper continues the study of reputation effects in games in which one player is engaged in "predatory conflict" with several opponents, each of whom is engaged only with the one. In line with the recent literature, we will call the single player the incumbent and its several opponents the entrants. We refer to the competition between the incumbent and one of its opponents as the basic contest. The notion of reputation enters into these games because each player is unsure about how its opponent(s) will play, and each uses the previous play by its opponent(s) to help predict future actions. Accordingly, in selecting current actions, each player must consider how this will affect not only immediate rewards but also the predictions, and hence future play, of its opponent(s). Using reputation as a loose synonym for those predictions, each player chooses current actions based in part on the reputation that will be built or maintained. Previous work (Kreps and Wilson (19S2), Milgrom and Roberts (1982)) showed that, in some situations, the incumbent's reputation would, as more and more entrants were added, come to dominate play of the game, if the

* Financial support from the following sources is gratefully acknowledged: National Science Foundation Grants SES84-08586 and SES85-09697, the Sloan Foundation, and the Institute for Advanced Studies at the Hebrew University. 
entrants were faced in sequence. ${ }^{1}$ Our aim is to see whether similar results obtain when the entrants are faced simultaneously. Along the way, we will also sharpen what is known about models in which opponents are faced sequentially.

The models that we examine yield two basic insights. First, the maintenance of a reputation in equilibrium depends on a tradeoff between the short run costs of maintaining that reputation (including the opportunity costs of not milking it most fully, if that would lead to its demise) and the longer run benefits that accrue from it. In the sequential contests models, the incumbent's short run cost is the cost of fighting a single entrant, while its long run benefits accrue from the many opponents to be faced in future contests. If the reputation has a positive value against each opponent, then as the number of opponents grows, the short run cost is eventually outweighed by the long run benefits. There is no similar effect on the incentives of the entrants to invest in reputation, and so the reputation of the single incumbent dominates.

The same basic tradeoff arises in simultaneous contest models, but with generally ambiguous results because the short run costs rise with the number of opponents. Long run benefits rise as well, but only when the long run benefits.rises more quickly than short run costs will we find that, as with sequential contests, the reputation of the one incumbent dominates. This happens in some formulations of simulaneous play, but it does not in others that are equally natural. The simple addition of opponents does not ensure that the reputation of the one will dominate, even when the net benefits per opponent of a particular reputation are positive. The reputation of the one only dominates if the structure of competition causes the long term benefits to outweigh short run costs. We will show how the ability of the entrants to reenter influences the direction of this comparison.

Second, the early literature might be interpreted as suggesting that incumbents generally favor conditions under which their reputations can (and do) dominate play. Compare the informationally linked situation above, in which each entrant observes earlier play in all contests, to a situation of informational isolation, in which each entrant observes only play in its own contest. (The comparison is between the weak incumbent's expected value from $N$ linked contests and its expected value in $N$ informationally isolated contests; not $N$

1 By "dominate play of the game," we mean that the incumbent will, in equilibrium, maintain its reputation regardless of how the entracts act, and so the entrants' equilibrium play is given by an easily solved optimization problem. 
linked vs. one isolated.) One might have thought that whenever the incumbent's reputation dominates linked play, the incumbent prefers linkage to isolation. Indeed, this is the case in the basic model of Kreps and Wilson (1982). But a small variation on their model (which is in the spirit of the basic model of Milgrom and Roberts (1982)) shows that this is not the case in general. Under linkage, the incumbent may defend his reputation because the short run costs of keeping it are outweighed by the long term opportunity losses that he would sustain if it were lost. Under isolation, he may be able to avoid the short run costs, and yet not suffer long term opportunity losses, the best of all possible worlds.

The paper begins, in section 2, with a very brief recapitulation of the basic model of the early literature. We present a formulation a bit more complex than in Kreps and Wilson and a bit less complex than in Milgrom and Roberts. This added complexity allows us to conclude section 2 with our first point: An incumbent might prefer informational isolation to informational linkage, even in cases in which the incumbent would fight to maintain its reputation.

In the early literature (and in section 2), the individual contest has a very simple temporal structure: First the entrant moves, and then the incumbent responds. Simultaneous contests of this sort would be uninteresting: If entrants made their (only) moves before the incumbent had to act, the incumbent's play could not influence the entrants' future actions, and so there would be no reputation effect. For this reason, we use a richer model of the individual contest, the concession game found in the back half of Kreps and Wilson. This is roughly a game of war of attrition, in which a player wins if its opponent concedes first. (If neither concedes, then both are losers.) Section 3 begins with a brief recapitulation of the equilibrium of the single contest if played in isolation. Then we look at sequences of these contests, and we confirm that the results from the early literature continue to hold. (Analysis of this latter point is consigried to an appendix.)

Sections 4 and 5 concern simultaneous play. In section 4 we analyze a formulation in which the reputation of the incumbent does not dominate play as the number of entrants increases. Instead, the number of entrants is irrelevant to the equilibrium outcomes. The key to the formulation in section 4 is that if and when the incumbent does concede, it retains the winnings it may have secured from earlier concessions by some of the entrants. In this case, the short run cost of maintaining a reputation is proportional to the number 
of entrants there are remaining, as are the reputation's long term benefits. Thus, changing the number of entrants has no effect on the nature of the equilibrium.

In contrast, in section 5 we formulate simultaneous play so that the incumbent, if it does ever concede, accrues no further benefits from contests it has already won. In the basic tradeoff, costs are proportional to the number of entrants who haven't yet conceded, while the gain from maintaining the reputation is proportional to the total number of all entrants. Thus there is the potential that benefits will exceed costs, and the incumbent's reputation will dominate. This does happen, for (at least) one specification of the model. In subsection 5.1, we present the basic model. In subsection 5.2 , we find the equilibrium for a simple case with two entrants. Even with two entrants, we are able to observe subtle effects brought about by linkage. In 5.3 we show how to compute the equilibrium for any number of entrants. Asymptotic results are obtained in subsection 5.4; these are in direct accord with those obtained when the individual contests are played in sequence.

Our use of terminology such as incumbent, entrant, simultaneous entry and reentry is meant to suggest applications to industrial organization, but the reader should not be misled by these terms into imagining that we intend the most obvious interpretions of them. In particular, when there is simultaneous entry, one naturally imagines a single market into which a number of entrants enter at once. One would expect in that situation that the actions of any one entrant would have a direct effect on the payoffs of other entrants. This would be an interesting game to investigate, but it is not the one that we examine. In the game considered here, each entrant's payoffs depend only on its own actions and those of the incumbent; other entrants effect it only insofar as their actions change the actions of the incumbent. The incumbent is concerned with its many opponents learning about it through actions it takes in other contests; entrants do not care what they reveal about themselves except to their single opponent. This is not to say that the entrants are not worried about their reputation, but that they are only concerned about their reputation with the single incumbent.

The models studied here only continue to scratch the surface of the reputation phenonmenon. In particular, a priori asymmetries in the characteristics of the entrants lead to many interesting effects. We hope to report on some of those effects in a sequel. 


\section{Sequential play with simple contest - a recapitulation.}

Kreps and Wilson (1982) and Milgrom and Roberts (1982) deal with models in which the individual contest has the following basic form. An entrant decides whether to enter or stay out of a particular market. If the entrant stays out, the contest ends. It is enters, the incumbent must react, either by fighting or acquiescing. With prior probability $1-p^{0}$, the incumbent's payoffs in the contest are: If the entrant stays out, the incumbent nets $a>0$; if the entrant enters and the incumbent acquiesces, the incumbent nets 0 ; if the entrant enters and the incumbent fights, the incumbent nets -1 . And with prior probability $p^{0}$, the incumbent has as strictly dominant strategy to fight any entry. For the entrant, with prior probability $1-q^{0}$, staying out nets 0 , entering and being met with acquiescence nets $b>0$, and being fought yields payoff -1 . And with prior probability $q^{0}$, the entrant does better to enter than to stay out, regardless of the incumbent's reaction. We refer to the incumbent as being weak if it has the first payoff structure; the incumbent is strong otherwise. And the entrant is weak or strong, if it has the first or second payoff structure, respectively. Since strong entrants and incumbents have dominant strategies, we will usually be lax in specifying equilibrium strategies and equilibrium expected payoffs, giving these for weak types of players only. ${ }^{2}$

The contest above has a unique sequential equilibrium. If there is entry, the incumbent acquiesces if weak, so a weak entrant nets expected payoff $\left(1-p^{0}\right) b+p^{0}(-1)$ if it enters. It enters, therefore, if $p^{0}<b /(b+1)$, stays out if the strict inequality is reversed, and is indifferent if there is equality. ${ }^{3}$

Now imagine that the incumbent faces a sequence of entrants, indexed by $n=N, \ldots, 1$. Entrant $N$ is faced first; after the contest against entrant $N$ is completed, $N-1$ is faced, and so on. Each entrant is weak with probability $1-q^{0}$, independent of the others. The incumbent is weak in all contests or strong in all of them, with prior probability $p^{0}$ that it is strong. For a strong incumbent, fighting in all markets where there is entry is strictly dominant. The weak incumbent's payoff total is the sum of payoffs in the $N$ contests, where payoffs in each contest are as above.

2 Kreps and Wilson (1982) consider the case where $q^{0}=0$. Milgrom and Roberts (1982) have a somewhat richer structure of payoffs, but the ones given here will suffice to make our points.

3 For the rest of the paper, we will ignore all such knife-edge cases. 
If the $N$ contests were conducted under conditions of informational isolation, where entrant $n$ does not observe the actions of the incumbent in earlier contests, then entrant $n$ would begin its contest with prior $p^{0}$ that the incumbent is strong, and a weak incumbent would acquiesce if there is entry. Hence weak entrants enter if and only if $p^{0}<b /(b+1)$. In this case, the weak incumbent nets zero in every contest; if $p^{0}>b /(b+1)$, then the weak incumbent nets an expected $\left(1-q^{0}\right) a$ per contest, or $N\left(1-q^{0}\right) a$ overall.

Things are much more complex if the $N$ contests are played under conditions of informational linkage, where entrant $n$ observes the past behavior of the incumbent. Then, if the incumbent acquiesces in contest $n$, it reveals that it is weak. This, it turns out, guarantees that all subsequent entrants will enter in at least one sequential equilibrium of the game. Hence the weak incumbent, in choosing actions in contest $n$, must weigh the short term costs of fighting $(-1)$ against the long term affect that acquiescence will have on its reputation, and hence on the entry decisions of subsequent entrants. From the early literature, we obtain the following result.

Proposition 1. When the incumbent faces $N$ entrants in sequential play of the simple contest, and the contests are informationally linked, there is a unique sequential equilibrium outcome. The nature of the equilibrium outcome for large $N$ depends on the sign of $q^{0}-a /(a+1)$.

(a) If $q^{0}>a /(a+1)$, then the incumbent, if weak, acquiesces at the first entry, which occurs "early" in the game. ${ }^{4}$ Hence as $N \rightarrow \infty$, the weak incumbent's average payoff per contest approaches zero.

(b) If $q^{0}<a /(a+1)$, then for every $p^{0}>0$ there is a number $n\left(p^{0}\right)$ such that, if there are $n\left(p^{0}\right)$ or more contests left to go, the incumbent is certain to fight any entry. Accordingly, weak entrants with label $n\left(p^{0}\right)$ or higher choose not to enter, and the weak incumbent's average payoff per contest approaches $\left(1-q^{0}\right) a-q^{0}$ as $N \rightarrow \infty$.

The early literature derived the unique equilibrium outcome, obtaining the asymptotic payoff functions as a corollary. A simpler argument is available for obtaining the asymptotic results, as developed in Fudenberg and Levine (1987). A synopsis of the simpler argument

4 If $p^{0}<b /(b+1)$, the first entry occurs in the first round of the game. If $p^{0}>b /(b+1)$, the first entry occurs at the earliest date that the entrant is strong. 
can be given here: Imagine that the incumbent follows the strategy of never conceding. Since the weak entrants will stay out unless the probability of being founght is sufficiently small, every time that a weak entrant enters and is fought the probability that the incumbent is strong must rise by an amount that is bounded away from zero. That is, each time the incumbent is called upon to invest in his reputation by a weak opponent (with positive probability), doing so must have a nonneglible effect on that reputation (measured by the posterior probability that the incumbent is strong). Thus there is a number $k$ independent of $N$ such that if there is positive probability of entry by $k$ (or more) weak entrants, then fighting by the incumbent leads to a reputation strong enough to deter all future weak entrants. This shows that the incumbent's asymptotic average payoff is bounded below by $a\left(1-q^{0}\right)-q^{0}$. If this expression is positive, then the bound is tight, since the incumbent must fight the strong entrants to have a positive average value asymptotically. In the case $a\left(1-q^{0}\right)-q^{0}<0$, then even if all weak entrants are deterred, the average value of fighting is negative. Hence the incumbent (if weak) will acquiesce early on, for an asymptotic average value of zero.

The case $q^{0}<a /(a+1)$ has received the most attention in the literature. Here the incumbent can credibly play the strategy "always fight" because the long-run consequences of acquiescence (entry by subsequent weak entrants) outweigh the short-run costs of fighting. To emphasize the crucial nature of the basic cost/benefit tradeoff, consider a formulation where the various contests have different sizes: Payoffs in the last contest (contest 1 ) are exactly the same as before. Payoffs in constest $n$ are $a+1$ times those in contest $n-1$; for example, in contest 2 the incumbent's cost of fighting is $a+1$, and his gain from deterring entry is $a(a+1) .{ }^{5}$ With these payoffs, it does not pay the incumbent to fight today, even if so doing deters all future entry. Thus, if $p^{0}<b /(b+1)$, for any $N$ the unique equilibrium is for all of the entrants to enter. This means that the incumbent can deter all future weak entrants by fighting, but the cost of doing so outweighs any conceivable future gain. It is the cost/benefit tradeoff, and not the frequency of play or the number of opponents per se, which is the key to understanding reputation effects.

When moving to a situation of informational linkage favors the incumbent's investment in reputation, we will say that the incumbent has gained in strategic backbone. Naturally,

5 One can also scale up the payoffs to the entrants, but this is clearly irrelevant. 
this stiffened backbone comes at a cost. The incumbent must fight all those that are not scared off which, in our simple model, amounts to all the strong entrants. $\dot{A}$ stiffened strategic backbone brings with it a loss of strategic flexibility. Milgrom and Roberts make clear that this loss of strategic flexibility may sometimes have a cost that outweighs the benefits from the stiffened backbone, so that the incumbent's reputation need not dominate play. This occurs when $q^{0}>a /(a+1)$. Moreover, even when the benefits of a stiffened backbone outweigh the costs of lost flexibility given that markets are linked, the (weak) incumbent may be worse off for having to make the choice.

Would the incumbent, if weak, prefer that the contests take place under conditions of informational linkage or informational isolation? Refer to figure 1.

When $q^{0}>a /(a+1)$ and $p^{0}<b /(b+1)$, the weak incumbent is indifferent between linkage and isolation. All the entrants enter if there is informational isolation, and it is too costly to maintain a stiff backbone with linkage.

When $q^{0}<a /(a+1)$ and $p^{0}<b /(b+1)$, informational linkage is preferred. Here the stiffened backbone deters some entrants, whereas none are deterred if the contests are informationally isolated, and the gain from a stiffened backbone outweighs the cost of maintaining it.

When $p^{0}>b /(b+1)$, in contrast, the weak incumbent strictly prefers informational isolation. The incumbent's ex ante reputation is sufficient to deter the weak entrants, so that moving from informational isolation to linkage (obtaining a stiffened backbone) provides no gains. The accompanying loss of strategic flexibility means, however, that the weak incumbent must fight all the strong entrants that come along, if it wants to keep its reputation. The weak incumbent either gives up the reputation altogether, if it costs too much to maintain (if $q^{0}>b /(b+1)$ ), or it maintains the reputation at the cost of fighting the strong entrants (if $q^{0}<b /(b+1)$ ).

The early literature focuses on the second case, where $p^{0}$ and $q^{0}$ are each small. This particular focus is natural if "strong" types are interpreted as "irrational" firms. But the case $q^{0}<a /(a+1)$ and $p^{0}>b /(b+1)$ should also be borne in mind. The first inequality ensures that, for large $N$, the incumbent's reputation dominates play of the game. If forced to choose between having a reputation for relative strength or having one for weakness, then the weak incumbent prefers strength. But the weak incumbent prefers most of all that it 
not have to prove itself, because it began a priori with a good reputation. It will fight, in equilibrium, but it does so more to avoid losing its good reputation and keep scared those who would be scared without linkage than to scare those who would not otherwise be.

\section{The Basic Concession Game}

The early literature makes use of a very simple contest, where the entrant moved once, and then the incumbent responded. To obtain interesting analyses of simultaneous play against many opponents, we need a contest in which the entrants have nontrivial responses to the incumbent's play. We use, for the rest of this paper, the concession game of Kreps and Wilson (1982, section 4) as the basic contest, and in this section we describe the game and its equilibrium. (Notation will be changed slightly from Kreps and Wilson (1982).)

There are two players, an incumbent and an entrant. The game is played in continuous time, over the interval from time one to zero. Time runs backwards. At each time $t$, if neither player has yet conceded to the other, either can choose to do so. As soon as one side or the other concedes, the game ends. If neither side concedes by time zero, the game ends.

As in section 2, each player is one of two types, strong or weak, with the prior probability that the incumbent [entrant] is strong being $p^{0}\left[q^{0}\right]$, independent of the type of the other player. A player's type is private information.

Payoffs to a player depend on which (if either) party concedes first, when the concession occurs, and the player's type. For strong players (both incumbent and entrant), it is a dominant strategy never to concede. As in section 2, then, we will be sloppy and sometimes drop the modifier "weak" in discussing the equilibrium strategies of the (weak) incumbent and entrant. For weak types of player, it is best to have one's opponent concede, second best to concede oneself, and worst to have an opponent that does not concede while not conceding oneself. Rewards and losses are proportional to the time periods over which they are received/incurred. Specifically, there are constants $a>0$ and $b>0$ with the following assignment of rewards:

Suppose the entrant concedes first, at time $t$. Thus the two fight for a period of length $1-t$, and the incumbent "wins" for a period of length $t$. The incumbent (if weak) nets 
reward

$$
\text { at }-(1-t)
$$

or $a$ per unit time that it wins, with a loss of -1 per unit time during the fight. And the entrant (if weak) nets $-(1-t)$, or zero per unit time after it concedes, and -1 per unit time during the fight. If the incumbent concedes first at time $t$, the (weak) entrant nets $b t-(1-t)$ and the (weak) incumbent nets $-(1-t)$. That is, the cost of fighting is minus one per unit time as before, conceding first nets zero for the remainder of the game to the side that concedes, and the entrant gets a reward of $b \cdot$ per unit time if the incumbent concedes first. If neither side concedes before time zero, then each loses a total of $-1 .^{6}$

In the equilibrium to this game, the two (weak) players engage in a game of "chicken." If neither concedes at the outset, each thereafter concedes at a "rate" until such time as the one or the other has concedes or each is certain that the other is tough. Precisely, suppose that at time $t$ neither side has yet conceded. Play to this time has led to (Bayesian) posterior reassessments concerning the toughness of the two players - $p_{t}$ will denote the probability that the incumbent is tough, and $q_{t}$ the probability that the entrant is tough. We hypothesize an equilibrium where the incumbent (if weak, and if it has not conceded by time $t)$ will concede over the period $(t, t-h)$ with probability $\pi\left(t, p_{t}, q_{t}\right) h$, up to terms that are $o(h)$, and the (weak) entrant with probability $\rho\left(t, p_{t}, q_{t}\right) h+o(h)$. (That is, $\pi$ and $\rho$ give hazard rates for the concession probabilities.)

Since the (weak) incumbent is (supposed to be) playing this mixed strategy in equilibrium, it must be indifferent between immediate concession at time $t$ and waiting until time $t-h$ and then conceding. The former nets zero for the remainder of the game. The latter has potential costs and benefits. With probability close to one, the entrant will not concede over this time period, and the costs will total $-h$. But there is a chance of order $h$, namely $\left(1-q_{t}\right) \rho h$, that the entrant does concede before time $t-h$. This involves the

6 It is important that rewards be proportional to the amount of time left. The analysis would change substantially if there were a fixed reward if the other side concedes first, regardless of when the concession takes place. Also, throughout this paper we will be less than perfectly formal. The reader may be troubled by our use of a continuous time game, for example. But note that this game can be viewed as a one-shot selection by each side of a "concession time" - a time at which one will concede if the other side hasn't done so first. This formulation is perfectly legitimate, and the analysis that follows describes an equilibrium for it. 
marginal probability that the entrant is weak $\left(1-q_{t}\right)$ times the conditional probability that the entrant will concede if weak $(\rho h)$. In this case, the benefit to the incumbent is at. Hence (up to terms of order $o(h)$ ) the weak incumbent is indifferent between the two strategies if and only. if

$$
0=-h+\left(1-q_{t}\right) \rho h a t \text {. }
$$

Dropping the $h$ and repeating the argument for the weak entrant gives the first two equilibrium conditions:

$$
\begin{gathered}
0=-1+\left(1-q_{t}\right) \rho a t \quad \text { and } \\
0=-1+\left(1-p_{t}\right) \pi b t .
\end{gathered}
$$

If the weak incumbent does use the strategy $\pi$, then $p_{t}$ will change through time. If ever there is a concession, this of course reveals that the incumbent is weak, and $p_{t}$ will drop to zero. But as long as there is no concession (and $\pi>0$ ), $p_{t}$ should rise. Simple application of Bayes' formula yields

$$
\dot{p}_{t}=p_{t}\left(1-p_{t}\right) \pi \quad \text { and } \quad \dot{q}_{t}=q_{t}\left(1-q_{t}\right) \rho
$$

(See Kreps and Wilson (1982). Here, $\dot{p}_{t}$ means $d p_{t} / d(-t)$, since time runs backwards.) Substituting (3.2) into (3.1) and dividing one equation by the other, we see that posteriors will evolve along a curve

$$
\frac{d q}{d p}=\frac{q b}{p a} \quad \text { or } \quad q=k p^{b / a}
$$

for some constant $k>0$.

We assert that $k=1$ is required if this is to give an equilibrium. Suppose, for example, that $k<1$. Then, tracing back through (3.1) and (3.2), one can see that, before time zero, $p_{t}$ will reach one at a time when $q_{t}=k<1$. Imagine that the incumbent (if weak) tries the strategy of no concession until just after this time. According to (3.1), the expected costs and benefits up to this time net out to zero. And at this time the entrant is convinced that the incumbent is tough. If weak (which happens with probability $1-k$ ), the entrant drops out immediately. Hence waiting until this time nets the (weak) incumbent a strictly positive expected value, and it could not be an equilibrium for it to use $\pi$. Similarly, $k>1$ is precluded. So, to have an equilibrium of the sort described, posteriors must evolve along 
the curve

$$
q=p^{b / a} \text {. }
$$

If $\left(q^{0}, p^{0}\right)$ falls along this curve, we do have an equilibrium. (To see this involves a little checking by the reader.) What if not? Refer to figure 2. If $\left(q^{0}, p^{0}\right)$ falls in the region labelled Region I, with $q^{0}$ "small" relative to $p^{0}$, then the game begins as follows: At time $T$, the entrant (if weak) concedes immediately with probability sufficient to make the posterior probability that it is tough if it doesn't concede equal to $\left(p^{0}\right)^{b / a}$. Thereafter, we follow the equilibrium described above. From region II, the (weak) incumbent concedes immediately with positive probability so that (once again) if there is no concession, the posteriors lie along the curve.

These strategies do give an equilibrium. If we lie in region I, for example, then the (weak) entrant randomizes at the start. This is a best response by the entrant: Dropping out nets zero for the entrant, as does continued fighting (as the posteriors begin to move along the curve).

Note that for each (weak) player, any positive expected value from the game is realized right at the start. After the initial randomization (if any), if the game hasn't ended, the expected value to both sides (if weak) is zero.

Using the methods of Fudenberg and Tirole (1986), one can show that this is the unique equilibrium of this game.

We introduce this concession game to study reputation effects when the incumbent plays many entrants simultaneously. But before doing so, we wish to record that sequential play of the concession game yields the same asymptotic results as does sequential play with the simple contests of section 2. Specifically, imagine that the incumbent faces a sequence of entrants, $n=N, N-1, \ldots, 1$. Each entrant is strong with probability $q^{0}$, independent of the others, while the incumbent is either strong in all contests or in none of them. Entrant $N$ is faced first, for the full unit of time, followed by entrant $N-1$, and so on. Under informational isolation, the equilibrium is simply $N$ copies of the equilibrium just described.

Equilibrium play of the $N$ contests under informational linlage is quite complex. We provide the sequential equilibrium of this game in the appendix, and we (only) record here that the incumbent's asymptotic payoffs are as with simple contests. 
Proposition 2. Imainge that the incumbent faces $N$ entrants in sequential play of the concession game.

(a) If $q^{0}>a /(a+1)$, the incumbent's average payoff per contest approaches zero as $N \rightarrow \infty$. Moreover, the incumbent (if weak) concedes "early" on in the game.

(b) If $q^{0}<a /(a+1)$, the incumbent's average payoff approaches $a\left(1-q^{0}\right)-q^{0}$. For "most" of the game, the incumbent will not concede whether weak or strong, so that weak entrants capitulate at the outset.

The appendix should be consulted for exact statements. We note also that the asymptotic average values can be derived more simply than in the appendix, using the general machinery of Fudenberg and Levine (1987).

The comparison of the payoff to the weak incumbent under conditions of informational isolation and linkage are also as before.

Proposition 3. (Refer to figure 3.)

(a) If $q^{0}>a /(a+1)$ and $p^{0}<\left(q^{0}\right)^{a / b}$, then the incumbent is asymptotically indifferent between linkage and isolation; under either regime, its expected value per contest has limit zero. (For any given $n$, since $v^{n} \geq 0$, the incumbent might favor informational linkage. But since $v^{\pi}<1$, the advantage from informational linkage is slight.)

(b) If $p^{0}>(a /(a+1))^{a / b}$ and $a /(a+1) \leq q^{0}<\left(p^{0}\right)^{b / a}$, then the incumbent asymptotically favors informational isolation. Indeed, under isolation the incumbent has a positive expected payoff per contest, whereas its expected payoff per contest falls to zero with informational linkage.

(c) If $p^{0}>(a /(a+1))^{a / b}$ and $q^{0}<a /(a+1)$, then the incumbent asymptotically favors informational isolation. The incumbent has a positive average payoff in either regime; in particular, its reputation dominates play for large $n$ under conditions of linkage. But it loses more from the loss of strategic flexibility than it gains from inducing more weak entrants to stay out for sure.

(d) If $p^{0}<(a /(a+1))^{a / b}$ and $q^{0}<a /(a+1)$, then the incumbent asymptotically favors informational linkage. The gains from stiffened strategic backbone outweigh the costs of lost strategic flexibility.

The proof is a simple matter of algebra, given proposition 2 , and it is left to the reader. 


\section{Simultaneous play with captured contests}

Now we turn to simultaneous play of the concession game. We imagine that one incumbent faces $N$ entrants in $N$ distinct contests which are played simultaneously, over the same unit length of time. As before, the incumbent is either tough in all contests or in none, and the entrants are either weak or tough, independent of each other and of the incumbent. Entrants observe what the incumbent does in all the contests and update their assessments accordingly. We restrict attention to equilibria in which the incumbent, if it concedes in any contest, must concede simultaneously in all contests in which the entrant has not yet conceded. (This is not imposed by restricting the strategies available to the incumbent. Rather, it will be equilibrium behavior in all the equilibria we examine.) Each entrant gets payoffs from its own contest; the incumbent's payoff is the sum of its payoffs in each contest.

Note well that when facing many entrants in sequence, the incumbent has a much longer horizon over which to amortize investment in its reputation than does any single entrant. Thus it is "natural" with sequential contests that, in some cases at least, the incumbent finds that the long-run benefits of a reputation outweigh its short-run costs, while the entrants find that reputation's costs outweigh its benefits. With simultaneous contests, the incumbent and the entrants amortize investments in reputation over the same horizon. Thus the effect of increasing the number of simultaneous entrants is not obvious a priori.

At this point, the specification of the simultaneous contest game is incomplete. What happens if some, but not all, of the entrants have conceded by date $t$, at which point the incumbent concedes? In equilibrium, the incumbent concedes in all the remaining active contests. But what happens in the contests that the incumbent has already won? (Note that this question is moot with sequential contests.) Does the (weak) incumbent continue to get the reward ( $a$ per unit time) from those contests it has already wor, or does it lose those flows of rewards for the time remaining? And what of the entrants that conceded previously? Do they continue to receive zero for the remainder of the game, or can they reenter to get $b$ per unit time for the time that remains?

In this section, we will complete the description of the game according to a no reentry scenario. The incumbent retains the rewards from contests already won, and entrants who 
conceded earlier get no benefit from the (subsequent) concession of the incumbent.

Proposition 4. In the no reentry scenario, the equilibrium strategies for the incumbent and the entrants are the same whether contests are linked informationally or are isolated. These strategies are independent of the number $N$ of entrants. The expected payoffs for weak entrants are precisely as in the one-vs.-one equilibrium of section 3, while the expected payoff to the incumbent is simply $N$ times the expected reward from the equilibrium of section 3.

In saying that the equilibrium strategies are the same, we mean that (after initial randomizations) each entrant uses the hazard rate $\rho$ defined in (3.1a), and the incumbent uses the hazard rate $\pi$ defined in (3.1b). Posteriors evolve along the curve $q=p^{b / a}$ (unless, of course, a player concedes). Initial randomizations are as in the one-vs-one equilibrium: In region I of figure 2, the (weak) entrants simultaneously and independently randomize at the start of the game, so that the posterior proability that each remaining entrant is tough is $\left(p^{0}\right)^{b / a}$, independent of the toughness of the others. In region II, the (weak) incumbent undertakes an initial randomization. ${ }^{7}$

All this follows from the equations analogous to (3.1). Suppose we reach a point where $k$ of the $N$ entrants have not yet conceded. The indifference equation for each entrant, which determines $\pi$, is unchanged from before. For the incumbent, things are slightly more complex. There are $k$ entrants to fight, so the rate of cost expenditure is $-k$. But there are $k$ entrants that might concede, and if each is conceding (independently) at hazard rate $\left(1-q_{t}\right) \rho$ (unconditional on type), then the hazard rate for the "next" concession is $k\left(1-q_{t}\right) \rho$, giving the incumbent a rate of expected gains equal to $k\left(1-q_{t}\right) \rho a t$. (Note here the use of the no-reentry scenario assumption that, if an entrant concedes, the incumbent is able to "bank" the amount at .) Thus the incumbent's indifference equation is

$$
0=-k+k\left(1-q_{t}\right) \rho a t
$$

which, cancelling the $k$, gives us the pair of equations (3.1). Both the cost (per unit time) and the expected benefits (fixing $\rho$ ) from continuing to fight rise linearly with $k$, so the

7 Of course, informational linkage "forces" the incumbent to concede, if at all, at times which are perfectly correlated among the contests. Under informational isolation, concession in the different contests could take place at, say, independently distributed times. 
equilibrating concession rate is unchanged with changes in $k$. Hence the old equilibrium continues to obtain. ${ }^{8}$

Without reentry, linking the contests does not stiffen the incumbent's backbone, regardless of $q^{0}$ and $N$. Contrast this with the previous section, where for small $q^{0}$ the incumbent's reputation dominates play for large $N$. There, increasing $N$ increased the (opportunity) cost of concession, but does not change the (momentary) cost of fighting. Here, both costs increase at the same rate. For the incumbent's reputation to dominate with simultaneous play, the cost of concession must (be able to) rise more quickly than does the number of entrants.

It is helpful to interpret this result using the "strategic backbone/strategic flexibility" notions introduced earlier. One gains strategic backbone only through the (potential) loss of strategic flexibility. That is, strategic flexibility is lost if one, for strategic reasons, would wish to treat a given opponent differently if others are watching from how that opponent would be treated if others could not observe. This translates to a gain in strategic backbone if this change in one's strategic reactions has salubrious effect on the initiating actions of others. Of course, to wish to treat one opponent differently from others, it is necessary that this opponent be somehow distinguished from the others. But in the no reentry scenario and the equilibrium we have constructed, entrants are distinguished only insofar as some have conceded and others have not. All those that have not conceded are identical. Since, without reentry, the incumbent need not be concerned with entrants that have already conceded, there is no loss in strategic flexibility. Hence there can be no gain in strategic backbone.

Imagine, however, that the game began with entrants that were tough with different probabilities. In particular, consider the case of two entrants, one of whom is almost sure to be strong, and the other is almost certainly weak. Without linkage, the incumbent would net almost a against the "weal" entrant and zero against the "strong" entrant, or $a$ in total. It should not be surprising that the incumbent does not do as well under conditions

8 Since we did not restrict the strategy space of the incumbent so that concession in all contests must take place simultaneously, we must say what happens should the incumbent concede in some, but not all, contests. At this point, entrants all assess probability one that the incumbent (i) is weak and (ii) will concede everywhere immediately, and the incumbent (if weak) does concede everywhere. The careful reader can chech that this out-of-equilibrium behavior supports the equilibrium we have given. 
of informational linkage, because it cannot afford to concede to the "strong" entrant until it has obtained concession from the weak. On the other hand, we believe, but have not shown, that if the parameters $a$ and $b$, as well as the prior, vary across contests, then the incumbent could gain by linkage. ${ }^{9}$

\section{Simultaneous contests with reentry}

\subsection{Two models}

In the sequential contests model we observed a loss of stategic flexibility, even though priors were equal, because in the course of play the incumbent could come to believe that its current opponent was more likely to be tough than are its future opponents. With simultaneous play, it is likewise the case that identical priors become unequal posteriors; viz., some entrants concede while the others fight. Without reentry this doesn't matter, because the incumbent needn't be concerned with entrants that have already conceded. But if entrants that have conceded can reenter, we can anticipate that the loss of strategic flexibility may be consequential, and that there may therefore be a gain from stiffened strategic backbone.

To investigate this, we consider here two variations on simultaneous play "with reentry." In both, if the (weak) incumbent ever concedes, then it nets a zero flow of benefits for the remainder of the game. That is, the incumbent loses benefits accrued from contests already won. The variations are distinguished in what happens to entrants that conceded prior to the incumbent's concession. In the first variation, called (hereafter) reentry scenerio $A$, all entrants receive reward at a rate of $b$ per unit time after the incumbent concedes, regardless of whether those entrants conceded earlier or not. In reentry scenario $B$, only entrants that have not already conceded receive this positive flow of reward. The description reentry is, therefore, more descriptive of scenario $A$ then it is of $B$; the reason for introducing scenario $B$ will become clear as we proceed.

The remainder of this section is organized as follows. In the next subsection, we analyze the equilibrium for two entrants and for $a>1$, both to provide a warm-up for the general case and to illustrate the tension between gain in strategic backbone and loss of strategic flexibility. In subsection 5.3, we present the equilibrium for general values of $N, a$ and

9 Cases of asymmetries of these and other sorts may be treated in a sequel. 
$b$. More precisely, we show how the equilibrium can be computed recursively; but we have not obtained closed form solutions. Finally, in subsection 5.4, we present analysis of the limiting form of the equilibria in 5.3, for large $N$, under the assumptions of scenario B. A summary of the results of subsection 5.4 are that for any $p^{0} \in(0,1)$, if $q^{0}>a /(a+1)$, then the incumbent (if weak) concedes at the outset with probability approaching one (proposition 4), while for $q^{0}<a /(a+1)$, all weak incumbents concede at the outset with probability approaching one (proposition 5 ). These results, once established, show that the (weak) incumbent's expected payoff per contest under scenario $B$ is precisely as in the case of opponents faced sequentially, and proposition 3 applies to simultaneous contests under scenario B, precisely as stated before for sequential contests.

\subsection{The case $N=2$ and $a>1$}

Consider the case of two entrants and $a>1$. If either of the two entrants concedes, then the (weak) incumbent's flow of payoffs is $a-1>0$ if the second entrant does not concede. Hence the concession of the first entrant ensures that the incumbent will never subsequently concede; fighting to the end dominates concession, no matter what the second entrant does. This in turn implies that if one entrant does concede and the remaining entrant is weak, the remaining entrant, knowing that the incumbent will now fight to the end, concedes immediately thereafter.

Accordingly, we derive an equilibrium of the following form. As in the case of a single entrant, we have a curve $q=f(p)$ that runs between $(0,0)$ and $(1,1)$, such that if there has been no concession yet, the incumbent is strong with some probability $p_{t}$, and each entrant is strong with probability $q_{t}=f\left(p_{t}\right)$, independent of each other and of the strength of the incumbent, then all the (weak) players have expected payofis for the rest of the game of zero. Each adopts a strategy of continuous randomization, the (weak) incumbent dropping with hazard rate $\pi$, and each entrant (if weak) dropping independently with hazard rate $\rho$, such that posteriors computed by Bayes' rule move up along the curve $(p, f(p))$. Before time zero is reached, either some player has conceded (which effectively ends the game), or it is known that all players are strong; that is, the posteriors $(1,1)$ are reached.

If, at the start of the game, $\left(p^{0}, q^{0}\right)$ is above the curve, then the (weak) incumbent randomizes between immediate concession and beginning to fight, such that no immediate concession causes the entrants to reassess the probability that the incumbent is strong to 
be $f^{-1}\left(q^{0}\right)$. And if $\left(p^{0}, q^{0}\right)$ lies below the curve, then the game starts with (independent) randomizations by the entrants (if weak) between concession and fighting such that, if they choose to fight, the probability that they are strong rises to $f\left(p^{0}\right)$. Note that, in this second. case, it is possible that each entrant is weak, and that after conducting these initial randomizations, one chooses to fight and the other chooses to concede. Then, since one entrant has conceded, the incumbent is sure never to concede, and the second entrant (because it is weak) concedes immediately. That is, a concession by one entrant at the start increases the chances of a concession by the second, although their strategies are chosen independently. This is a manifestation of continuous time, where we are imagining that one player can react instantaneously to the actions of second; the phenomenon is just as in Fudenberg and Tirole (1985). ${ }^{10}$

To specify the equilibrium, it remains to give $\pi$ and $\rho$, which will also identify the curve $q=f(p)$. Analysis just as in section 4 is employed. Each entrant, while employing a continuous randomization strategy $\rho$, must be indifferent between immediate concession (at time $t$ ) and concession after $h$ more units of time. The cost of waiting the extra $h$ units is (with probability very close to one) $-h$. There is probability $\left(1-\dot{p}_{t}\right) \pi h$ that the incumbent will concede, which will confer a benefit of $b t$. And there is probability $\left(1-q_{t}\right) \rho h$ that the other entrant will concede; should this occur, the first entrant (if weak) will concede immediately, for a zero continuation value. Since immediate concession by this entrant means that the incumbent will never concede, concession either at $t$ or $t-h$ means a continuation value of zero (in either scenario A or B), and the entrant's indifference equation, which yields $\pi$, is

$$
0=-1+\left(1-p_{t}\right) \pi b t
$$

For the incumbent's indifference relation, note first that if each entrant (if weak) is conceding at rate $\rho$ independently of the other, and each is weak with probability $1-q_{t}$

10 The reader may be concerned with our less than completely formal style, especially since the general theory of games in continuous time is underdeveloped. A completely tight formalization of all the analysis we give in this section is possible, using one of two game forms. In the first, periods of "real time", the lengths of which are determined by the minima of stopping times chosen independently by players, are interspersed with periods of discrete, fictitious time, in which players react to each others' actions. In the second, players choose sets of stopping times, where, in particular, each entrant chooses a time at which it will concede if $k$ or more of its fellows entrants have conceded, for each $k=0, \ldots, N-1$. We leave all these formal details to the interested reader. 
independently of the other, then the rate of the "next" concession by an entrant is the sum of the individual rates, or $2\left(1-q_{t}\right) \rho$. Thus we have the indifference equation

$$
0=-2+2\left(1-q_{t}\right) \rho\left(a+a\left(1-q_{t}\right)-q_{t}\right) t
$$

The terms on the right hand side are the cost of fighting (divided by $h$ ) -2 , and then the rate of the next concession $2\left(1-q_{t}\right) \rho$ by an entrant times the expected gain to the incumbent if one of the two concedes, $\left(a+a\left(1-q_{t}\right)-q_{t}\right) t$. This last term is the gain at from the one entrant who does concede, plus the expected gain $\left(a\left(1-q_{t}\right)-q_{t}\right) t$ from the second entrant.

Equations (5.1) are substituted into Bayes' rule (3.2) and integrated with the boundary condition that the curve $q=f(p)$ passes through $(1,1)$. This gives the curve, with $p$ written as a function of $q$,

$$
p=q^{2 a / b} e^{(1+a)(1-q) / b} .
$$

In figure 4 we graph this curve together with the curve for a single entrant, $p=q^{a / b}$. The reader can easily verify that the picture is as we have shown, with the curve for two entrants lying below the curve for one for high values of $q$, and lying above for low values of $q$.

We can see in this picture the tension between the two sides of the informational linkage of the two contests, the beneficial stiffened strategic backbone and the costly loss in strategic flexibility. For low values of $q$, gains from stiffened strategic backbone exceed losses from the loss of strategic flexibility, because if one entrant can be made to concede, it is likely that the stiffened strategic baclbone will cause the other to concede. For high values of $q$, if one entrant concedes there are good prospects that the other will have to be fought to protect the gains aiready won, so the losses from lost strategic flexibility outweigh the gains from stiffened backbone.

We should note that a comparison of informational linkage vs. informational isolation in this case is not entirely trivial. If $\left(p^{0}, q^{0}\right)$ lies above both curves in figure 4 , then the (weak) incumbent nets zero in either case. If the initial data lie in the vertically crosshatched region, linkage gives the incumbent a positive expected payofi, while isolation gives expected payoff zero in each contest, so that linkage is clearly preferred. Similarly, it is clear that isolation is preferred in the horizontally cross-hatched region. But in the region below both curves, where the (weak) incumbent has a positive expected payoff in either regime, 
the exact computations must be carried out. We will spare the reader the details and simply make the assertion that at points $\left(p^{0}, q^{0}\right)$ below both curves, and where $p^{0}$ is at or below the level at which the two curves intersect, informational linkage is preferred. But for $p^{0}$ above the intersection level, there are points $\left(p^{0}, q^{0}\right)$ below both at which informational isolation is preferred.

\subsection{Construction of an equilibrium for the general case}

The equilibrium of this game for the general case has a relatively simple form, but it is quite difficult to compute precisely. We will begin by giving the form of the equilibrium, and then we will show how, inductively, one would compute it.

Let $K$ be the smallest integer such that $(N-K) a-K \geq 0$ and $(N-K-1) a-(K+1)<$ 0 . That is, $K=\lfloor N a /(a+1)\rfloor$, where $\lfloor\cdot\rfloor$ denotes "integer part of". Note that $K=0$ is possible. To keep matters simple, we will assume that $(N-K) a-K>0$.

Description of the equilibrium. In either scenario $A$ or $B$, the game has an equilibrium of the following form. (Refer to figure 5.) For each $k=K+1, \ldots, N$, there is a corresponding curve in the unit square state space, passing through $(0,0)$ and $(1,1)$, with the curve for $k$ lying above the curve for $k+1$.

(i) At every time in the game, there is identical probability that any entrant is strong, and the strength of different entrants are independent and independent of the strength of the incumbent. We denote the (common) probability that an entrant is strong by $q_{t}$, and the probability that the incumbent is strong by $p_{t}$.

(ii) If the initial datum $\left(p^{0}, q^{0}\right)$ lies above and to the left of the $N$ curve, then the game begins with an immediate randomization by the weak incumbent between concession and beginning to play, such that, conditional on no concession, the probability that the incumbent is strong rises to take us to the $N$ curve.

(iii) If at any time in the game (including the start), there are $k>I$ entrants that have not previously conceded, and if $\left(p_{t}, q_{t}\right)$ lies below and to the right of the $k$ curve, then those of the $k$ "remaining" entrants that are weak randomize independently between immediate concession and continuing to play, so that (for each) the posterior probability of strength given no concession moves to the $k$ curve. Note well that if we begin with, say, 
$k$ entrants, and all randomize, there is positive probability that some number $j<k$ will continue to fight and $k-j$ will concede immediately. If $j>K$, then we will again be at a point below the relevant curve (now the $j$ curve), and a subsequent randomization between immediate concession and continuing to fight will be required. This "cascade" of instantaneous randomizations ends in one of two ways: Either there are some some number $k^{\prime}>K$ of entrants remaining, and the posterior probability of their strength is such that $\left(p_{t}, q_{t}\right)$ lies along the $k^{\prime}$ curve; or there are $K$ or fewer entrants remaining.

(iv) If at any time in the game there are $K$ or fewer entrants remaining, then the incumbent, strong or weak, will fight to the end with probability one. Accordingly, of the remaining $K$ entrants, all that are weak will concede immediately.

(v) If at any time in the game there are $k>K$ entrants remaining, and the state of the game lies along the $k$ curve, then the entrants and incumbent concede with hazard rates that cause the state of the game to move along the $k$ curve. This behavior ends one of three ways: The point $(1,1)$ is reached (which will happen before time runs out in the game). The incumbent concedes, in which case the game is over. One entrant concedes, in which case, as we are below the $k-1$ curve, the prescription of step (iii) above is followed.

The equilibrium described has as basis the following consideration: If ever $N-K$ or more of the entrants have conceded, then the weak incumbent will never concede; the positive flow from the $N-K$ contests won is enough to cover the cost of fighting the $K$ contests remaining, even if none of the $K$ remaining ever concede. (In terms of the case $N=2$ and $a>1, K$ is one - as soon as the first entrant concedes, the incumbent is sure to fight for the rest of the game.) Hence, at any point at which the incumbent faces $K$ or fewer live entrants, all the remaining entrants that are weat will concede immediately. It is the prospect of such an event that keeps the incumbent fighting, and (in equilibrium) the incumbent's instantaneous cost of fighting must just be covered by the instantaneous expected value composed of the chance that such an event will occur times the value the incumbent will receive for the remainder of the game if the event occurs. The concession rates of live entrants are adjusted in equilibrium so that this equation holds. And the concession rate of the incumbent is set so that live entrants are just compensated for fighting by the (instantaneous) chance that the incumbent will concede. Equations similar in spirit 
to (3.1) (and (5.1)) govern concession rates, and hence generate the curves that describe the equilibrium.

The exact equations that replace (3.1) are a good deal more complex than (3.1), on two grounds. Consider first the indifference equation of the incumbent, if there are $k$ entrants alive, each conceding with hazard rate $\rho$. If the incumbent concedes, it will net zero for the remainder of the game, hence the left hand side of the replacement for (3.1a) remains zero. The instantaneous cost of fighting is $k$. The rate at which the next concession takes place is $\left(1-q_{t}\right) k \rho$. But, whereas in (3.1a) (and in (6.1)) the next concession nets at for the incumbent, here it does not. The expression for the incumbent's expected value, given this initial concession, is a monster: The $k-1$ remaining entrants all randomize immediately, with concession probability that would carry the posterior up to the $k-1$ curve (whose placement is derived in the previous step of a recursive derivation). Conditional on the number of the $k-1$ entrants that do not concede (unless it is all $k-1$ ), we now have another immediate randomization to carry posteriors up to a curve of still lower index, etc. Working through all these, we get a marginal distribution for (i) the probability that this cascade of randomizations ends on a $j$ curve for $K<j<k$ with $j$ entrants still live, in which case the incumbent nets expectation zero in continuation, and (ii) the probability that it ends with $j \leq K$ live entrants. If it ends with $j \leq K$ live entrants (the others all strong with some probability $q^{*}$ that depends in a nontrivial way on $j)$, the incumbent nets an expected reward of $t\left[a\left((K-j)+j\left(1-q^{*}\right)\right)-j q^{*}\right]$. (This is at from each of the $K-j$ already dead entrants, plus at from the each of $j$ remaining that concede immediately, less $-t$ from each of the $j$ remaining that turn out to be strong.) If we have the placement of the $j$ curves for all $j<k$, then this expected gain is certainly computable, step by step. But it seems rather formidable analytically.

As for the analogue to (3.1b), the distinction between scenarios $A$ and $B$ becomes important. Scenario B is the easier to deal with. If an entrant concedes, it will net zero for the remainder of the game. Hence the left hand side of the analogue to $(3.1 b)$ is zero. The instantaneous cost of fighting is -1 , as in (3.1b). And the instantaneous expected gain arises from the chance that the incumbent concedes, which occurs at rate $\left(1-p_{t}\right) \pi$ and nets $b t$ for the entrant. (If some other entrant concedes, either the game continues along some other $j$ curve, along which the entrant nets zero expected value. Or the game 
ends with a cascade leading to $K$ or fewer entrants, which will mean a payoff of zero for a (weak) entrant.) Hence, in scenario $\mathrm{B}, \pi$ is given by precisely $(3.1 b)$.

In scenario $\mathrm{A}$, things are more complex. Note that a weak entrant who has conceded does not necessarily have zero expected payoff for the remainder of the game. If there are more than $K$ entrants left, there is a chance the incumbent will concede, and then the entrants that conceded earlier can reenter to accrue a positive reward. Hence the value of immediate concession, which goes on the left hand side of the analog to $(3.1 b)$, is no longer zero. Rather, it is the solution of an integral equation. The right hand side of the entrant's indifference equation will include instantaneous cost (incurred at rate 1) and the gain $b t$ times the probability that the incumbent concedes, as before. But also, at rate $(k-1)\left(1-q_{t}\right) \rho$, some other entrant might concede, and this will effect the value to the weak entrant whose payoffs we are attempting to balance. The exact impact will depend, as before, on the distribution of the outcome of the cascade of randomizations let loose by any concession (although now we must condition that distribution on the knowledge of the one entrant that it, at least, is indeed weak) and on the value of continuation to a weak entrant for each of those outcomes. Finally, since this is meant to evaluate the expected value to an entrant of conceding after $h$ more time units, we must compute that (no longer zero) expected value, which involves yet another set of probabilities of how the next cascade will resolve. Once again, the curves are computable in theory (although now they are integral instead of differential equations).

One sees from the this description how to prove that an equilibrium of the form given above does exist. For $k=K+1, \ldots, N$, one "computes" the $k$ curve recursively, with the curves for $j<k$ already in place. The important step in the recursion is to show that the $k$ curve lies below the $k-1$ curve. To see that this is so, note that in the analogue to $(3.1 a)$, as the $k$ curve comes closer to the $k-1$ curve, the probability that all $k-1$ entrants continue to fight after an initial concession goes to one. Hence the "gain" term for the weak incumbent goes to zero. To achieve equality in the analogue to $(3.1 a), \rho$ must increase, which increases the slope of the $k$ curve, pushing it away from (and below) the $k-1$ curve. (The exact argument is a bit more complex in scenario $\mathrm{A}$, owing to changes in the analogue to (3.1b), but we will not go into details.) An equilibrium exists that does have the form given, and, with a large computer budget, one could solve (to any desired 
level of accuracy) for the position of the curves.

\subsection{Asymptotic results}

While we are unable to obtain a closed form solution for the equilibrium, we are able, in scenario $\mathrm{B}$ at least, to obtain asymptotic results for large $N$. As in the literature on sequential contests, the comparison of $q^{0}$ and $a /(a+1)$ is the key. Let us point out, however, that here the asymptotics do not follow from the general argument of Fudenberg and Levine (1987), as no matter how many entrants are involved, the incumbent and the entrants have the same decision horizon. That is, the reputation of each will be of value for the same length of time. Of course, the incumbent's reputation is of value against more opponents, but we know from the no-reentry case that this fact alone is not decisive. What matters is, of course, the comparison of costs and benefits of the reputation.

Throughout this section, we let $K_{N}$ denote $[a N /(a+1)\rfloor$, and we will assume that $a$ is irrational, so that $a N /(a+1)$ is never itself integer.

Proposition 4. In scenario B, if $q^{0}>a /(a+1)$, then as $N \rightarrow \infty$ the incumbent begins the game by conceding with probability approaching one.

Proof. The idea of the proof is that, for $q^{0}>a /(a+1)$, as $N$ goes to infinity there is vanishingly small probability that more than $N-K_{N}$ entrants will ever concede, because there is vanishingly small probability that more than $N-K_{N}$ entrants are in fact weak. Thus there is vanishingly small chance that the incumbent will ever get a net positive flow of reward (in fact, we'll show that the expected reward varishes with $N$ ), so the incumbent will only fight for a very small period of time. But since, in scenario $B$, the amount of time the incumbent must be willing to fight is the time it takes $p_{t}$ to reach one, and the equation for the evolution of $p_{t}$ is independent of $N$, it must be that $p_{1}$ (after initial randomizations) is, in the limit, one. For any fixed $p^{0}$, this gives the result of the proposition.

A bit more formally, note that in the equilibrium, it is a best response for the incumbent to refuse to concede until such time as $\left(p_{t}, q_{t}\right)$ reaches $(1,1)$ (where, of course, the incumbent does not concede if, before this time, $N-K_{N}$ or more entrants do in fact concede). The time $\tau_{N}$ at which $p_{t}$ reaches 1 in the game with $N$ entrants, depends on 
the probability $p_{1}^{N}$ that the incumbent is strong after any initial randomizations and the differential equation $\dot{p}=p / b t$. In particular, this time approaches 1 (that is, the amount of time that elapses until $(1,1)$ is reached approaches zero) in $N$ if and only if $p_{1}^{N}$ goes to 1 . So we aim to show that $\tau_{N}$ must approach one as $N \rightarrow \infty$.

In the game with $N$ entrants, if the incumbent waits until time $\tau_{N}$, then the chance that the incumbent "wins" before time $\tau_{N}$ is precisely the probability that there are $K_{N}$ or fewer strong entrants initially. If there are more than $K_{N}+1$ strong entrants, then the incumbent will, by waiting until $\tau_{N}$, lose at least $(a+1)\left(1-\tau_{N}\right)$ (and, probably, a good deal more). If there are precisely $K_{N}$ strong entrants, the incumbent will lose some amount. And if there are $K_{N}$ or fewer strong entrants, then $a N$ is the most the incumbent can hope to win. Thus, by following the "don't concede until $\tau_{N}$ " strategy, the ex ante expected payoff to the incumbent is bounded above by:

\section{$\operatorname{Prob}\left(K_{N}\right.$ or fewer entrants are strong $) \times a N$}

- Prob(more than $K_{N}+1$ strong entrants) $\times(a+1)\left(1-\tau_{N}\right)$.

From Feller (1968, VII.6), the first probability goes to zero at a rate exceeding $\exp \left(-N^{1 / 4}\right) / N^{1 / 6}$ and the second probability goes to one. (The estimate given is fairly crude.) Hence for the expected value to be nonnegative, it must be that $\tau_{N} \rightarrow 1$, which completes the proof.

Proposition 5. In scenario B, if $q^{0}<a /(a+1)$, then as $N$ approaches $\infty, N-K_{N}$ or more entrants concede at the outset (and the incumbent "wins") with probability approaching one.

Proof. We will not give all the details, although the reader should be able to reconstruct them from the following sketch. To begin, establish some notation and terminology. Let

$$
\lambda=\left(\frac{a+1}{a} q^{0}+1\right) / 2 .
$$

By assumption, $\lambda$ is less than one. Imagine that the incumbent, if weak, plays the strategy of no concession until such time as posteriors $(1,1)$ are reached. Note that this happens at some time $t^{*}>0$, which can be bounded below by integrating the equation for the 
evolution of $p_{t}$ with the initial condition $p_{1}=p^{0}$. (It is at this point only that we use the assumption that scenario B pertains; to ensure that the law of motion of $p_{t}$ does not change with $N$. Note that if the game begins with a randomization by the incumbent, $p_{1}$ will exceed $p^{0}$, and $t^{*}$ will be larger.)

Imagine this incumbent watching the game evolve as he plays this particular waiting strategy (which, we note, is among his best responses). Let $\kappa(t)$ be the stochastic process that gives the number of remaining entrants at time $t$, and let $\phi(t)$ denote the (common) posterior probability that the remaining entrants are strong. For definiteness, we fix the right continuous versions of these stochastic processes: $\kappa(t)$ gives the number of remaining entrants after any cascade of randomizations at time $t$, etc. We will use $\kappa\left(t^{-}\right)$and $\phi\left(t^{-}\right)$ to denote the left continuous versions. Let $S(t)$ and $S\left(t^{-}\right)$be equal to $\kappa(t) \phi(t)$ and $\kappa\left(t^{-}\right) \phi\left(t^{-}\right)$, respectively. That is, $S(t)$ is the expected number of strong entrants given the information revealed up to (and including) time $t$.

Now consider two stopping times. The first is the first time (if it occurs) that there are $K_{N}$ or fewer entrants remaining - the first hitting time of $\left\{\kappa(t) \leq K_{N}\right\}$. The second is the first time that $\phi(t) \kappa(t) / N \geq \lambda a /(a+1)$. Note that, for large $N$, one of $\tau_{1}$ or $\tau_{2}$ must occur prior to $t^{*}$. The first stopping time marks a time at which the incumbent has "won". The second marks a time at which the remaining entrants are surprisingly strong. The meaning of the second description arises from the following result:

As $N \rightarrow \infty$, the probability that $\tau_{1}$ occurs before $\tau_{2}$ approaches one.

We leave the proof for later, but the intuition should be clear. Given the prior probability $q^{0}$ that any entrant is strong, the expected number of strong entrants subsequently, $S(t) / N$, must have expected value $q^{0}$. Moreover, for large $N$, there can be little variance in its value. So the probability that $S(t) / N$ exceeds $\lambda a /(a+1)$ must approach zero as $N \rightarrow \infty$.

Now suppose that at some time $t$, the incumbent "wins"; that is, the time $\tau_{1}$ is reached (before $\tau_{2}$ ). We claim that the expected number of strong entrants, conditional on this event, is bounded above by $S\left(t^{-}\right)$. To see this, note that $S\left(t^{-}\right)$is the expected number of strong entrants just before time $t$. The probability of enough concessions at time $t$ to cause the "win" (given that at time $t$ one of the $k\left(t^{-}\right)$did concede), conditional on the number of actually strong entrants, is decreasing in the number of strong entrants. 
Hence the expected number of strong entrants, conditional on a win at time $t$, is less than the unconditional expectation $S\left(t^{-}\right)$. Thus the conditional expected value to the incumbent, conditional on a win at time $t$ and conditional on $\kappa\left(t^{-}\right)$and $\phi\left(t^{-}\right)$, is at least $\left(a N-(a+1) S\left(t^{-}\right)\right) t^{*}$. Since $\tau_{2}$ was not hit before time $t, S(t) / N<\lambda a /(a+1)$, and we have a lower bound on this conditional expected value of $a N(1-\lambda) t^{*}$.

At time $t$, before the times $\tau_{1}$ and $\tau_{2}$, the maximal rate of cost to the incumbent is $N$. Until these two stopping times, in our equilibrium the instantaneous cost to the incumbent must just equal the hazard rate at which the incumbent "wins" times the conditional expected value it receives if it does win. So we see that the hazard rate of a win for the incumbent at times before $\tau_{1}$ and $\tau_{2}$ is bounded above, uniformly in $N$, by $1 /\left(a(1-\lambda) t^{*}\right)$. But then by integrating the hazard rate, we see that, over the time interval from time one to time $t^{*}$, the chance of a "no win" (that $\tau_{1}$ is delayed until after $\tau_{2}$ ) is bounded below by

$$
\operatorname{Prob}\left(\text { no win at outset) } \times e^{-\left(1-t^{-}\right) /\left(a(1-\lambda) t^{*}\right)}\right. \text {. }
$$

If we know the probability of "no win before $\tau_{2}$ " must go to zero in $N$, then probability of a win at the outset must go to one.

To complete the proof, it remains to establish that the probability that $\tau_{1}$ is less than $\tau_{2}$ goes to one as $N \rightarrow \infty$. To show this, note that the maximum of $\tau_{1}$ and $\tau_{2}$ is a stopping time, and that $S(t) / N$ is a bounded martingale, so that $E(S(\tau) / N)=q^{0}$, where $\tau=\max \left(\tau_{1}, \tau_{2}\right) \cdot{ }^{11}$ Fix any $\epsilon>0$, and let

$$
\delta=\epsilon\left(\frac{a}{a+1}-q^{0}\right) / 4
$$

Select $N$ sufficiently large that

$$
\text { Prob(fraction of strong entrants } \left.<q^{0}-\delta\right)<\delta \text {. }
$$

(That sufficiently large $N$ do exist follows from the estimate given in the proof of the previous proposition.) Now decompose $E(S(\tau) / N)$ into three pieces: The integral over the set $\Lambda_{1}$ where $\tau_{2}>\tau_{1}$; the integral over the set $\Lambda_{2}$ where $\tau_{1}>\tau_{2}$ and the fraction of strong entrants is $q^{0}-\delta$ or less; and integral over the set $\Lambda_{3}$ where $\tau_{1}>\tau_{2}$ and the 11 Recall that clocks run backwards in this model, so that the maximum of two stopping times is a stopping time, etc. 
fraction of strong entrants is above $q^{0}-\delta$. The integral over $\Lambda_{1}$ is bounded below by the probability of $\Lambda_{1}$ times $\left(q^{0}+a /(a+1)\right) / 2$, from the definition of $\Lambda_{1}$. The integral over $\Lambda_{2}$ is nonnegative. And the integral over $\Lambda_{3}$ is bounded below by $q^{0}-\delta$ times its probability. Hence $E(S(\tau) / N)$ is bounded below by

$$
\begin{gathered}
\operatorname{Prob}\left(\Lambda_{1}\right) \times\left(q^{0}+\frac{a}{a+1}\right) / 2+\operatorname{Prob}\left(\Lambda_{3}\right) \times\left(q^{0}-\delta\right) \\
\geq q^{0} \times\left(\operatorname{Prob}\left(\Lambda_{1}\right)+\operatorname{Prob}\left(\Lambda_{3}\right)\right)-\operatorname{Prob}\left(\Lambda_{3}\right) \delta+\operatorname{Prob}\left(\Lambda_{1}\right)\left(\frac{a}{a+1}-q^{0}\right) / 2 \\
\geq q^{0}-q^{0} \times \operatorname{Prob}\left(\Lambda_{2}\right)-\delta+\operatorname{Prob}\left(\Lambda_{1}\right)\left(\frac{a}{a+1}-q^{0}\right) / 2 \\
\geq q^{0}-2 \delta+\operatorname{Prob}\left(\Lambda_{1}\right)\left(\frac{a}{a+1}-q^{0}\right) / 2 .
\end{gathered}
$$

By the definition of $\delta$, then, the original integral can equal $q^{0}$ only if the probability of $\Lambda_{1}$ is less than $\epsilon$, which completes the proof.

Although it takes a bit more work, the methods used to prove propositions 4 and 5 can be used to describe the location of the $k$ curves $\left(k>K_{N}\right)$ for large $N$. Fixing $p \in(0,1)$, for $\alpha \leq a /(a+1)$, the $q$ level of the $\lfloor\alpha N\rfloor$ curve at $p$ (in the $N$ entrant game) approaches $a /(\alpha(a+1))$. That is, the picture is as in figure 6 .

In both propositions, we needed to assume that scenario $B$ prevailed. In the first proposition, we needed to know that the motion of $p_{t}$ does not become very quick (from any given starting point) in $N$; that if $p_{t}$ has to reach 1 in a short period of time, then it would have to begin at a value close to 1 . In the second proposition, we needed to know that the motion of $p_{t}$ does not become very slow; that starting from $p^{0}$ (or above), when the posterior 1 is reached, there is still some amount of time (bounded below in $N$ ) left in the game. So it seems clear that one of the two propositions must hold in scenario A. Intuition would suggest that the incumbent is better off in scenario $A$ than in $B$. In scenario A, entrants face a "free rider" problem in that it is better for any single entrant to sit on the sidelines and let the other entrants fight the incumbent than to fight itself. If the incumbent does concede, an entrant on the sidelines gets all the benefits (in scenario A) that accrue to one who fought. If this intuition is correct, then proposition 5 would be the one more likely to survive in scenario A. 
In fact, we believe that both propositions do in fact survive in scenario $\mathrm{A}$. A earlier version of this paper (available upon request) shows that this is so at the "limit" game, in which the incumbent faces a continuum of entrants. But scenario $B$ is sufficient to make the point that we wish to make, namely that with simultaneous play, one must look closely at the structure of the game to see if the reputation effect of the incumbent dominates. We leave further analysis to the interested reader.

\section{References}

Feller, William, An Introduction to Probability Theory and Its Applications, Vol. 1 (3rd edition), John Wiley and Sons, New York, 1968.

Fudenberg, Drew and David Levine [1987], "Equilbirium Selection in Repeated Games with a Single Long-lived Player Facing many Short-lived Opponents," mimeo.

Fudenberg, Drew and Jean Tirole [1986], "A Theory of Exit in Oligopoly," Econometrica, in press.

[1985], "Preemption and Rent Equalization in the Adoption of New Technology," Review of Economic Studies, Vol. 52, 383-402.

Kreps, David and Robert Wilson [1982], "Reputation and Imperfect Information," Journal of Economic Theory, Vol. 27, 253-279.

Milgrom, Paul and John Roberts [1982], "Predation, Reputation, and Entry Deterrence," Journal of Economic Theory, Vol. 27, 280-312. 


\section{Appendix: Sequential play of the concession game}

In this appendix, we analyze sequential play of the concession game, as outlined at the end of section 3. In particular, we provide a proof of proposition 2. This is done for a particular equilibrium, which we will construct along the way. (We do not know that this is the only sequential equilibrium of this game, although we believe that it is.) We use the following notation: the expected value to the weak incumbent from equilibrium play in contests $n, n-1, \ldots, 1$ if contest $n$ beings with the entrants assessing that the incumbent is strong with probability $p$ is denoted by $v_{n}(p)$. (Recall that contests are indexed backwards, so that contest 1 is the last.) The probability $q^{0}$ enters as a parameter to this function and is held fixed throughout. We will prove the following strengthened version of Proposition 2.

\section{Proposition $2^{\prime}$.}

(a) In the equilibrium to this game, $v_{n}(p)$ is nondecreasing in $p$.

(b) If $q^{0}>a /(a+1)$ then $v_{n}(p)<a\left(1-q^{0}\right) / q^{0}<1$, and the long run expected value per contest to the (weak) incumbent approaches zero. There is a constant $k\left(p^{0}\right)$ (independent of $N$ ) such that the incumbent, if weak, will fight for a total length of time that is bounded above by $k\left(p^{0}\right)$. Hence the probability that the incumbent (if weak) has not conceded by the end of round $N-m$ decays exponentially in $m$.

(c) If $q^{\theta}<a /(a+1)$, then there is a number $n\left(p^{0}\right)$ such that in all contests against entrants of index greater than $n\left(p^{0}\right)$, the incumbent (weak or strong) will fight for the full unit time with certainty. Accordingly, weak entrants in those contests concede immediately. And $\lim _{N \rightarrow \infty} v_{N}(p)=a\left(1-q^{0}\right)-q^{0}$ (for all $\left.p^{0}>0\right)$.

\section{The equilibrium}

The first step is to give the equilibrium construction. Recall that the incumbent, if strong, will always fight, and strong entrants always enter. Hence we will describe only the equilibrium strategies of the weak incumbent and weak entrants. Throughout, $q^{0}$, the prior probability (at the beginning of each stage) that the current entrant is strong is treated as a parameter and is suppressed in the notation. We use $v_{n}(p)$ to denote the expected payoff to the weak incumbent for play of the game from stage $n$ to stage 1 , inclusive, when stage $n$ begins with the entrants assessing probability $p$ that the incumbent is strong.

The equilibrium takes the following form at stage $n$ :

(o) If, at any point in the game, the incumbent concedes, whether in equilibrium or out, entrants revise assessments that the incumbent is strong to be zero. Thereafter, the incumbent concedes immediately in all contests, and entrants never concede.

(i) If $v_{n-1}(p) \geq 1$, then the (weak) incumbent will refuse to concede in the current round, 
even if convinced that the current entrant is strong. Hence weak entrants will concede at the outset of this round. It is immediate in this case that $v_{n}(p)=a-(a+1) q^{0}+v_{n-1}(p)$. (ii) If $v_{n-1}(p)<1$, then there is a curve analogous to the curve $q=p^{b / a}$ along which play evolves in this stage, after initial randomization by one side or the other which takes us to the curve. The placement of this curve depends on $n$ and on $p$. It is given by indifference equations analogous to (4.1), which in this case are

$$
1=\left(1-p_{t}\right) \pi b t \text { and } 1=\left(1-q_{t}\right) \rho\left(a t+v_{n-1}\left(p_{t}\right)\right),
$$

where $t$ in these equations refers to the time remaining in the current stage. These indifference equations, together with Bayes's rule (4.2), give differential equations

$$
\dot{p}=\frac{p}{b t} \quad \text { and } \quad \dot{q}=\frac{q}{a t+v_{n-1}(p)}
$$

for the evolution of the posteriors in the current stage. The boundary condition for this curve (analogous to the condition in the single contest game that the curve passes through $(1,1)$ ) is: At the time $t^{*}$ (in the current stage) at which $q_{t^{*}}=1$ (the entrant is certain to be tough), the value to the incumbent beginning the next stage must satisfy the inequality

$$
v_{n-1}\left(p_{t^{*}}\right) \leq t^{*}
$$

If (A.3) holds as a strict inequality, then the boundary condition is that $p_{t^{*}}=1$, and the weak incumbent's strategy has called for him to concede with probability one by this point. If (A.3) holds as an equality, then $p_{t^{-}}<1$ is possible, and the weak incumbent's strategy calls for him to refuse to concede for the remainder of the current stage.

We establish that an equilibrium of this form exists by induction on $n$, the number of stages remaining in the game. We will show, as we proceed, that the boundary condition given in (ii) is uniquely specified as we compute $v_{n}$ recursively. As part of the induction hypothesis, we establish that each $v_{n}$ has the following properties:

(iii) $v_{n}$ is nonnegative, has value zero at $p=0$, is nondecreasing and strictly increasing when it is nonzero, and it is continuous.

\section{Initiating the induction.}

As the first stage in our inductive proof, we note that $v_{1}$, the value function associated with the equilibrium for a single stage of the game given in section 3 , has all the properties given in (iii). Thinking of $v_{0}$ as being identically zero, we see as well that the equilibrium for this last stage is described by (ii). This initiates the induction.

If the incumbent ever concedes 
We note next that ( 0 ) above is indeed compatible with (sequential) equilibrium. As long as entrants will never again concede, the weak incumbent wishes to concede immediately in all cases. Entrants, expecting the incumbent to concede immediately with probability one (and being unshakeable in this belief) will never concede. Note that this shows that $v_{n}(0)$ is indeed zero. Also, since a strong incumbent will never concede, the beliefs entailed in ( 0 ) are consistent with any equilibrium strategies for this game.

\section{Beginning with $v_{n-1}(p) \geq 1$}

Suppose the properties above have been established for all $j \leq n-1$. Consider play beginning at the start of stage $n$, with initial probability $p^{n}$ that the incumbent is strong. If $v_{n-1}\left(p^{n}\right) \geq 1$, then play as described in step (i) is certainly equilibrium play. If the incumbent will not concede at all in this stage, then weak entrants concede immediately. If weak entrants concede immediately, then the incumbent will be certain, if there is no concession, that the current entrant is strong, and hence the current stage will cost 1 . Given ( 0 ), if the weak entrant does concede, then $p$ will move to zero, and the incumbent will then net the continuation value $v_{n-1}(0)=0$. Hence it is an equilibrium for the weak incumbent to pay the cost of 1 and get in return the continuation value $v_{n-1}\left(p^{n}\right)$.

(Indeed, this is the only possible path of equilibrium play at this stage, given that $v_{n-1}$ gives the continuation value. If in some equilibrium the weak incumbent were to concede with positive probability, then Bayes' rule would force beliefs given concession to zero, giving zero continuation value next period. Since fighting will lead to a $p^{n-1}$ beginning the next stage which is no lower than than the current value $p^{n}$, and since $v_{n-1}$ is (by the induction hypothesis) strictly increasing above $p^{n}$ (and, hence, is strictly greater than one), it cannot be an equilibrium for the weak incumbent to concede with positive probability.)

This implies that $v_{n}\left(p^{n}\right)=a-q^{0}(a+1)+v_{n-1}\left(p^{n}\right)$. Thus $v_{n}$ inherits all the properties of $v_{n-1}$ that are outlined in (iii) for $p$ above the smallest value such that $v_{n}(p) \geq 1$.

\section{Beginning with $v_{n}(p)<1$ - preliminaries}

The harder case is where we begin stage $n$ with $p^{n}$ such that $v_{n-1}\left(p^{n}\right)<1$. Note that, in such circumstances, the behavior described in (i) is not equilibrium behavior. If the entrants play as in (i), then unless the entrant concedes immediately, the incumbent concludes that he faces a strong entrant, but the value of continuation if he follows the equilibrium strategy and does not concede is insufficient to compensate him for the cost of fighting to the end of the current period.

We construct instead an equilibrium of the form outlined in (ii). There is, as in section 3 , an initial randomization by one side or the other, such that if there is no initial concession, then both sides (if weak) have continuation value zero for the rest of the game. Each then concedes continuously, until such time as either one or the other concedes, or until an appropriate boundary condition is reached. We use, as before, $\pi$ to denote the concession 
(hazard) rate of the weak incumbent, and $\rho$ to denote the concession rate of the weak entrant. By the same sort of logic as in the one stage game, $\pi$ and $\rho$ must satisfy the indifference equations ( $A .1$ ) given above. The equation for $\pi$ is just as before - the weak entrant must just be compensated for the cost of fighting by the expected rate at which the incumbent will concede, times the prize won if the incumbent does concede. The equation for $\rho$ has an additional term, since concession by the entrant gives the weak incumbent at in the current contest and $v_{n-1}\left(p_{t}\right)$ beginning the next. Bayes' rule is as always, so in this regime of continuous randomizations, posteriors evolve according to the differential equations $(A .2)$.

$v_{n}(p)<1$ - solutions of the differential equations and the $\tau$ function

The next step is to consider the solution to the differential equations $(A .2)$ as a function of initial conditions. Refer to figure A1. We suppose that we start the current round at some point $\left(p^{n}, q^{0}\right)$, and that there may be an initial randomization by one (weak) side or the other, so the differential equations will be initiated at some point $\left(p_{1}, q_{1}\right)$ with either $p_{1} \geq p^{n}$ and $q_{1}=q^{0}$ or $p_{1}=p^{n}$ and $q_{1} \geq q^{0}$. That is, we begin the differential equations running at some point along one of the two rays emanating from $\left(p^{n}, q^{0}\right)$ that are shown in figure A1. Given the assumptions about $v_{n-1}$ in the induction hypothesis, the solutions to the differential equations are well behaved in their initial conditions:

(iv) The solutions to the differential equations $(A .2)$, as functions of their initial conditions along the rays shown in figure $A 1$, are continuous in those initial conditions. Paths from two different initial conditions do not intersect.

The equation for $p_{t}$ integrates to show that, since $p_{1} \geq p^{N}$, as long as $p^{N}>0$, the level $p_{t}=1$ is reached by some time prior to time zero. Hence from each of the possible initial conditions along the two rays, there is a time $t^{*}$ depending on the initial conditions at which the path of $\left(p_{t}, q_{t}\right)$ exits from the unit square. Moreover, because of (iv), there is a continuous bijection between initial conditions along the two rays and "terminal positions" $\left\{(p, 1): p \geq p^{n}\right\} \bigcup\left\{(1, q): q \geq q^{0}\right\}$ at which $\left(p_{t}, q_{t}\right)$ departs the unit square.

Accordingly, there is some particular initial condition $(\hat{p}, \hat{q})$ along one of the two rays such that the solution of (A.2) starting from that point passes out of the unit square at $(1,1)$. Figure $A 1$ is drawn so that $(\hat{p}, \hat{q})$ lies to the right of $\left(p^{r}, q^{0}\right)$; this need not be the case in general. However the following is generally true. For every $p^{\prime} \in\left[p^{0}, 1\right]$, there is a unique starting position $\left(p\left(p^{\prime}\right), q\left(p^{\prime}\right)\right)$ lying to the right and/or above $(\hat{p}, \hat{q})$, along one of the two rays, such that with $\left(p\left(p^{\prime}\right), q\left(p^{\prime}\right)\right)$ as starting position, the solution to the differential equations exits the unit square at $\left(p^{\prime}, 1\right)$. These initial conditions are continuous in $p^{\prime}$.

Let $\tau\left(p^{\prime}\right)$ be the time remaining when the curve that exits at $\left(p^{\prime}, 1\right)$, starting at $\left(p\left(p^{\prime}\right), q\left(p^{\prime}\right)\right)$, hits $\left(p^{\prime}, 1\right)$. Being excessively formal, $\tau\left(p^{\prime}\right)$ is such that, in the solution 
to $(A .2)$ with initial condition $\left(p\left(p^{\prime}\right), q\left(p^{\prime}\right)\right), p_{\tau\left(p^{\prime}\right)}=p^{\prime}$ and $q_{\tau\left(p^{\prime}\right)}=1$. (Note that $p\left(p^{\prime}\right), q\left(p^{\prime}\right)$, and $\tau\left(p^{\prime}\right)$ all depend on the initial point $\left(p^{n}, q^{0}\right)$, since $\left(p^{n}, q^{0}\right)$ determines the two rays that form the set of possible initial conditions.) Now we can show:

(v) For any initial point $\left(p^{n}, q^{0}\right), \tau\left(p^{\prime}\right)$ is continuous and strictly decreasing in $p^{\prime} \in\left[p^{n}, 1\right]$. Moreover, for fixed $p^{\prime}, \tau\left(p^{\prime}\right)$ is nonincreasing in $p^{n}$, and it is strictly decreasing if $p\left(p^{\prime}\right)=$ $p^{n}$.

We leave to the reader the task of establishing (v), using the differential equations (A.2) and the assumed monotonicity (in $p$ ) of $v_{n-1}$. Note that $\tau\left(p^{n}\right)=1$ and $\tau(1)>0$.

\section{$v_{n-1}(p)<1-$ terminal conditions and the equilibrium}

We can now derive the terminal condition that determines the equilibrium beginning at stage $n$ with $p^{n}$. Refer to figures A2(a) and A2(b). We have graphed there $\tau\left(p^{\prime}\right)$ and $v_{n-1}\left(p^{\prime}\right)$ for $p^{\prime} \in\left[p^{n}, 1\right]$. Note that, by assumption, $v_{n-1}\left(p^{n}\right)<1=\tau\left(p^{n}\right)$. Hence we either have a picture as in A2(a), in which the two curves intersect once and once only at some $p^{\prime} \in\left(p^{n}, 1\right]$, or we have the picture in A2(b), in. which $v_{n-1}(1)<\tau(1)$. The uniqueness and existence of an intersection point (if $v_{n-1}(1) \geq \tau(1)$ ) follows from the facts that both $v_{n-1}$ and $\tau$ are continuous, $\tau$ is strictly decreasing, and $v_{n-1}$ is strictly increasing (where it is nonzero).

Imagine that $v_{n-1}(1)<\tau(1)$. Then we use as terminal condition for stage $n$ the condition that the curve must pass through the point $(1,1)$. At this point, by assumption, the value to the weak incumbent starting in the next stage is less than the cost the incumbent would pay to wait for the next stage to begin (since the incumbent now knows that the current entrant is strong with probability one). Accordingly, if the weak incumbent waits until this posterior is reached, its optimal continuation is to concede immediately, netting value zero. This is the right terminal condition; as in the single contest analysis, we can now integrate back from this terminal condition to show that the weak incumbent's expected payoff is zero all along the curve through $(1,1)$, hence $\pi$ is a best response for the weak incumbent to its opponents' strategies.

Now consider the case where the curves $v_{n-1}$ and $\tau$ do intersect. We use in this case the terminal condition that the curve for stage $n$ passes through the point $\left(p^{*}, 1\right)$, where $p^{*}$ is the point of intersection of the two curves. By construction, when the time $\tau\left(p^{*}\right)$ is reached, the value of continuation beginning in the next stage is just equal to the cost the weak incumbent must incur to get to that stage. Hence the value to the incumbent at this terminus is zero. Once again, the incumbent's value all along the curve integrates back to be identically zero, and the incumbent is using a best response to its opponents' strategies.

It is important that, in the second case, the incumbent does not concede at the time $\tau\left(p^{*}\right)$ with positive probability - it continues on to the next stage with probability one. 
Of course, after the instant $\tau\left(p^{*}\right)$ the incumbent wishes strictly to remain, as the value beginning the next stage is undiminished, while the cost remaining to be paid to get to that stage decreases as time passes. But at $\tau\left(p^{*}\right)$, the incumbent is just indifferent. Still, if the incumbent were to concede with positive probability at $\tau\left(p^{*}\right)$, then the weak entrant would, by waiting until (just after) $\tau\left(p^{*}\right)$, obtain a positive expected value, contradicting the optimality of the strategy specified by $\rho$.

\section{$v_{n-1}(p)<1-$ completing the induction step}

We have now established that the equilibrium in stage $n$ has the form we outlined. To complete the induction (and finish the proof that there is an equilibrium of this form), we must show that $v_{n}$ inherits all the required properties. Continuity of $v_{n}$ is tedious but straightforward: If $p^{n}$ moves a bit, so do the associated initial conditions $p\left(p^{\prime}\right)$ and $q\left(p^{\prime}\right)$ for each $p^{\prime}$, and (therefore) so does $\tau\left(p^{\prime}\right)$. The intersection point $p^{*}$ therefore moves a bit, which means that the initial condition at the intersection point $q\left(p^{*}\right)$ moves continuously in $p^{n}$, and this initial condition continuously determines $v_{n}$.

Nonnegativity of $v_{n}$ is obvious. It remains to show that $v_{n}$ is strictly increasing in $p^{n}$ when it is nonzero. Fix, therefore, some $p^{n}$ such that $v_{n}\left(p^{n}\right)>0$, and consider $v_{n}\left(\hat{p}^{n}\right)$ for some $\hat{p}^{n}>p^{n}$. The argument was given forr the case $v_{n-1}\left(p^{n}\right) \geq 1$. So we may suppose that $v_{n-1}\left(p^{n}\right)<1$. Since $v_{n}\left(p^{n}\right)>0$, we know from the form of the equilibrium that the appropriate starting condition for the corresponding curve is $\left(p^{n}, q\right)$ for some $q>q^{0}$, and the weak incumbent's expected payoff is $v_{n}\left(p^{n}\right)=\left(1-q^{0} / q\right)\left(a+v_{n-1}\left(p^{n}\right)\right)$. Consider two cases. First, if $v_{n-1}\left(\hat{p}^{n}\right) \geq 1$, then $v_{n}\left(\hat{p}^{n}\right)=a-(a+1) q^{0}+v_{n-1}\left(\hat{p}^{n}\right) \geq$ $a+v_{n-1}\left(\hat{p}^{n}\right)-\left(a+v_{n-1}\left(\hat{p}^{n}\right)\right) q^{0}=\left(1-q^{0}\right)\left(a+v_{n-1}\left(\hat{p}^{n}\right)\right)>v_{n}\left(p^{n}\right)$. The other possibility is the $v_{n-1}\left(\hat{p}^{n}\right)<1$, so that $v_{n}\left(\hat{p}^{n}\right)=\left(1-q^{0} / \hat{q}\right)\left(a+v_{n-1}\left(\hat{p}^{n}\right)\right)$, where $\hat{q}$ is the posterior appropriate to starting at $\left(\hat{p}^{n}, q^{0}\right)$. By the monotonicity of $v_{n-1}$, we have the desired result as soon as we show that $\hat{q} \geq q$.

This follows from the derivation of terminal conditions in these cases: As noted before, the $\tau$ curve for the starting value $p=\hat{p}^{n}$ is (as a function of the terminating $p^{\prime}$ ) everywhere greater than the $\tau$ curve for the starting value $p=p^{n}$. Thus the equilibrating $p^{*}$ and the amount of time left $\tau$ must both be greater starting in $\hat{p}^{n}$ than in $p^{n}$. But if $\hat{q}<q$, the argument used to prove (vi) above can be used to show that $\tau$ will be less for $\left(\hat{p}^{n}, \hat{q}\right)$ than for $\left(p^{n}, q\right)$, a contradiction. Thus we have an equilibrium of the form described.

\section{The equilibrium for large $N$}

We next characterize the equilibrium for large $N$. It is easiest to begin with the case $q^{0}>a /(a+1)$, for which the following holds:

(vi) If $q^{0}>a /(a+1)$, then $v_{n}(p) \leq a\left(1-q^{0}\right) / q^{0}<1$, uniformly in $p$ and $n$. Accordingly, the equilibrium behavior is never of the form described in (i) but always has the form (ii). 
There is an upper bound $k\left(p^{0}\right)$ on the amount of time the incumbent (if weak) will fight, and (therefore) the probability that the incumbent (if weak) has not conceded by the end of round $N-m$ decays exponentially in $m$.

We establish the uniform bound on $v_{n}$ by induction. In the last stage, $v_{1}(p)<a\left(1-q^{0}\right)$ which is less than our bound. Suppose that it has been shown that $v_{n-1}<a\left(1-q^{0}\right) / q^{0}<1$, uniformly in $p$. Then we know that the form of the equilibrium in stage $n$, regardless of $p^{n}$, has the form (ii), and the expected payoff to the weak incumbent is $\left(1-q^{0} / q\right)\left(a+v_{n-1}\left(p^{n}\right)\right.$, which is bounded above by $\left(1-q^{0}\right)\left(a+a\left(1-q^{0}\right) / q^{0}\right)=a\left(1-q^{0}\right) / q^{0}$. (An easier proof is to use the monotonicity of $v_{n}$ in $p$ and compute directly $v_{n}(1)$.)

(Indeed, one can show that for any $p<1$ this bound is not tight: $\lim _{N \rightarrow \infty} v_{N}(p)<$ $a\left(1-q^{0}\right) / q^{0}$. This in turn implies that no matter how large is $N$, the probability that the incumbent, if weak, concedes in the first contest is bounded away from zero. We leave this to the reader.)

To obtain the uniform bound on the total amount of time that the weak incumbent will fight, reason as follows. If ever $p$ reaches one, by that time the incumbent, if weak, must have conceded with probability one. Hence we wish to show that $p$ reaches one after the incumbent has fought for an amount of time that is bounded above (depending on $p^{0}$ ). We can obtain such a bound from the law of motion of $p$, since any time $p$ is moving, $\dot{p} \geq p / b$. The proof is complicated slightly by the fact that the weak incumbent may be fighting at times when $p$ is not moving. (Recall that when the state reaches. the boundary $q=1$, the incumbent does not concede for the remainder of the contest.) But in any contest in which the incumbent does fight at all, $p$ must be moving for the first $1-a\left(1-q^{0}\right) / q^{0}$ units of time; if the boundary was struck before that time, then the time remaining would exceed the continuation value, in contradiction to the nature of the equilibrium (unless $p$ has already reached one). So for every one unit of time that the incumbent fights, $p$ moves for $1-a\left(1-q^{0}\right) / q^{0}$ or more. This, together with a bound on how long $p$ can be moving before it strikes one, gives an upper bound on how long the (weak) incumbent will fight.

Finally, the incumbent, if weak, will have to fight every strong entrant it meets, for the full unit of time (until it concedes). Given an upper bound $k\left(p^{0}\right)$ on the amount of time the weak incumbent will fight, the chance that it will live beyond the first $k\left(p^{0}\right)+m$ contests is bounded above by the chance that in those first $k\left(p^{0}\right)+m$ contests, $m$ or more of the entrants faced are weak. This clearly decays exponentially.

Large $N$ and $q^{0}<a /(a+1)$

When $q^{0}<a /(a+1)$, a very different picture emerges.

(viii) If $q^{0}<a /(a+1)$, then there is a number $n\left(p^{0}\right)$ such that in all contests of index greater than $n\left(p^{0}\right)$, the incumbent (weak or strong) will fight for the full unit of time with 
certainty. That is, the equilibrium behavior is as in (i) above. Accordingly, weak entrants in those contests concede immediately. And $\lim _{N \rightarrow \infty} v_{N}(p) / N=a-(a+1) q^{0}$.

First recall from the previous step that we can easily bound the total amount of time that the weak incumbent randomizes between fighting and conceding, irrespective of $N$ (depending on $p^{0}$ ), since whenever such randomization is taking place, the evolution of $p_{t}$ is governed by the relationship $\dot{p}=p / b t \geq p / b$.

Suppose that we have $n$ contests left to play in the game. We will develop a bound on the expected length of time that there will be fighting, if the (weak) incumbent adopts the (nonequilibrium) strategy of never conceding, and the entrants follow their equilibrium strategies. ·

$$
\begin{aligned}
& \left.E \text { [total time fighting] }=\sum_{k=1}^{n} E \text { [time fighting in contest } k\right]= \\
& \sum_{k=1}^{n} E \text { [time fighting in contest } \mathrm{k} \text { and entrant } \mathrm{k} \text { is tough] }+ \\
& E \text { [time fighting in contest } \mathrm{k} \text { and } \mathrm{k} \text { is weak }]= \\
& \left.\sum_{k=1}^{n} q^{0}+\sum_{k=1}^{n} E \text { [time fighing in contest } \mathrm{k} \text { and } \mathrm{k} \text { is weak }\right] \leq \\
& n q^{0}+K .
\end{aligned}
$$

The last inequality follows from the previous paragraph - the total time fighting weak opponents is bounded by $K$, because whenever the opponent is weak and is fighting, the probability $p$ must be moving.

Hence by following the strategy of fighting all the way to the end, starting with $n$ stages left and against the equilibrium strategies of the entrants, the weak incumbent will net an expected value of

$$
a E \text { [time not fighting] }-E \text { [time fighting] }=a n-(a+1) E \text { [time fighting] }
$$

which, by the previous estimate, is no less than

$$
n\left(a-(a+1) q^{0}\right)-(a+1) K
$$

For $n$ sufficiently large, under the hypothesis that $q^{0}<a /(a+1)$, this exceeds one. Since the optimal response by the weak incumbent must do at least this well, we know that for all sufficiently large $n, v_{n}\left(p^{0}\right)>1$, which is what we wanted. The remainder of (viii) follows trivially. 


\section{$v_{n}$ is nondecreasing in $n$}

To complete the analysis, we establish that $v_{n}$ is nondecreasing in $n$. If $v_{n-1}\left(p^{n}\right) \geq 1$, then $v_{n}\left(p^{n}\right)=a-(a+1) q^{0}+v_{n-1}\left(p^{n}\right)$. Moreover, the condition $v_{n-1}\left(p^{n}\right) \geq 1$ requires that $q^{0}<a /(a+1)$. Hence we have the result.

If $v_{n-1}\left(p^{n}\right)=0$, then the result is trivial. Hence we have left the case where $0<$ $v_{n-1}\left(p^{n}\right)<1$. Now assume inductively that $v_{n-1} \geq v_{n-2}$. Looking at the differential equations $(A .2)$, we see that for any termination point $\left(p^{\prime}, 1\right)$, the curve for stage $n-1$ will be more steeply sloped than the curve for stage $n$. Moreover, looking at figure A2, the $\tau$ function for stages $n$ and $n-1$ are the same, at least in the neighborhood and to the left of the solution for $n-1$, since (as $v_{n-1}\left(p^{n}\right)>0$ ) we know that the time left upon hitting $\left(p^{\prime}, 1\right)$ from $\left(p^{n}, q\right)$ depends on the evolution of $p$, the differential equation for which is independent of $n$. Combined with the induction hypothesis that $v_{n-1} \geq v_{n-2}$, this means that the equilibrating terminal condition in stage $n$ is moved leftward from the terminal condition for stage $n-1$. Accordingly, for the same starting point $\left(p^{n}, q^{0}\right)$ in stages $n$ and $n-1$, the relevant curve in stage $n$ lies everywhere above the curve for $n-1$. At stage $n$, then, there is higher probability that the entrant will concede immediately. And if the entrant does concede immediately, the incumbent receives a larger reward (from the value of continuation). Thus $v_{n}>v_{n-1}$. 


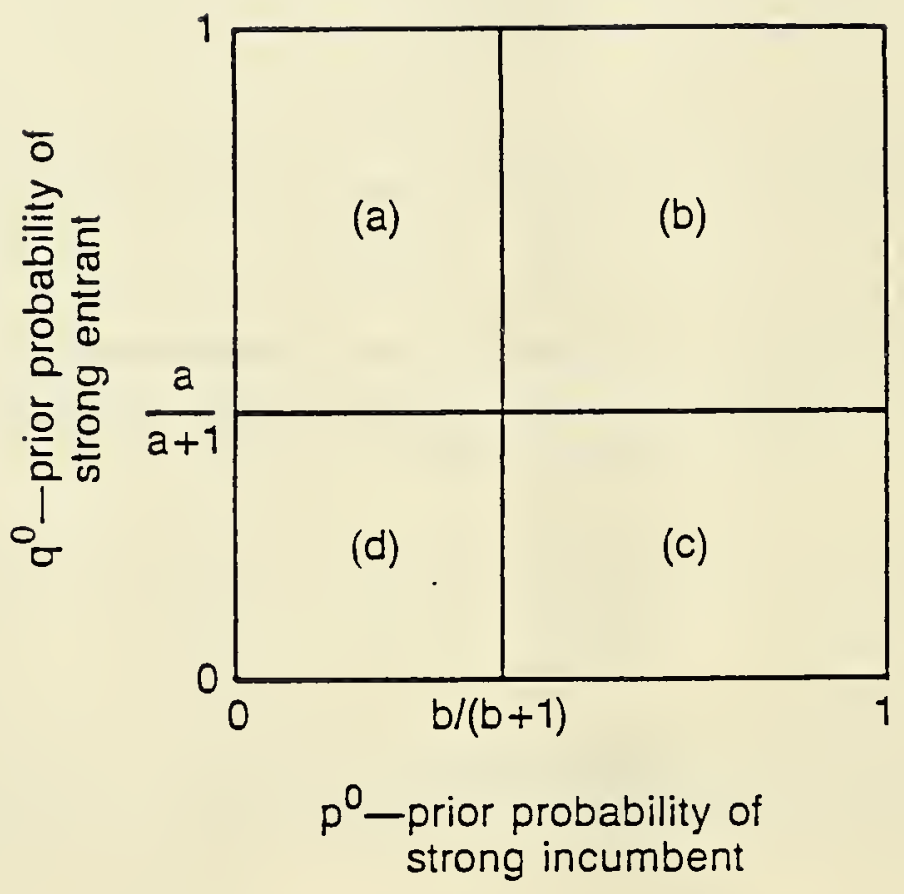

Figure 1. Isolation vs. linkage with the simple contest. With informational linkage, the weak incumbent's asymptotic payoff per contest is zero in regions (a) and (b), and $a-(a+1) q^{0}$ in regions (c) and (d). With isolation, the asymptotic payoffs are zero in regions (a) and (d) and $a\left(1-q^{0}\right)$ in regions (b) and (c). Hence the weak incumbent prefers linkage in region (d), isolation in (b) and (c), and is indifferent between isolation and linkage in region (a). 


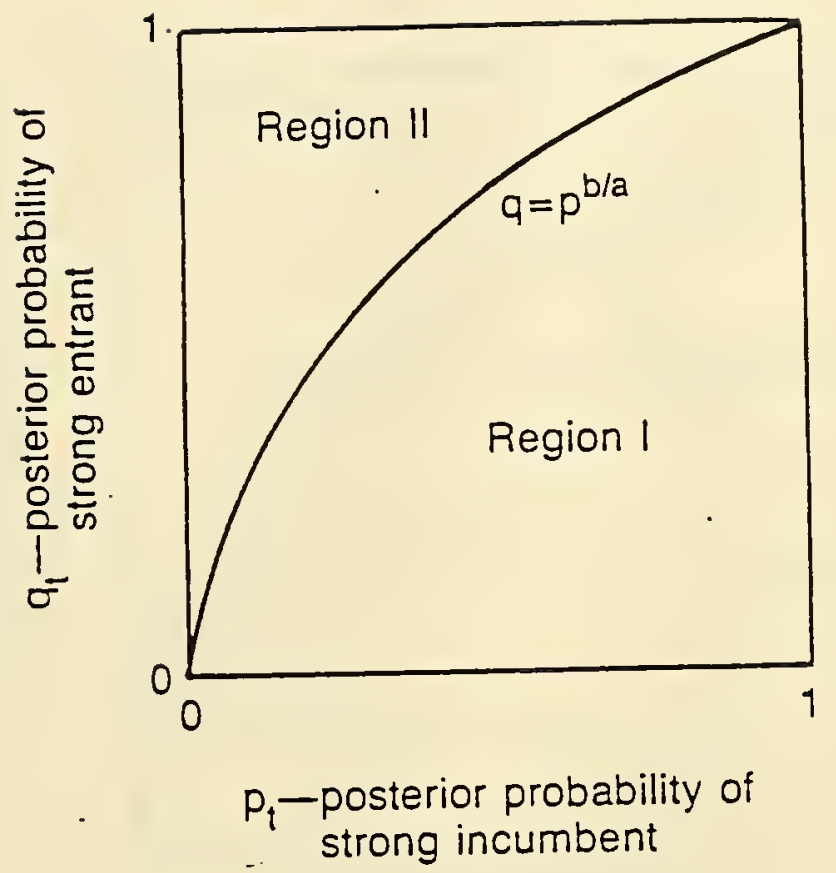

Figure 2. State space for the concession game contest. 


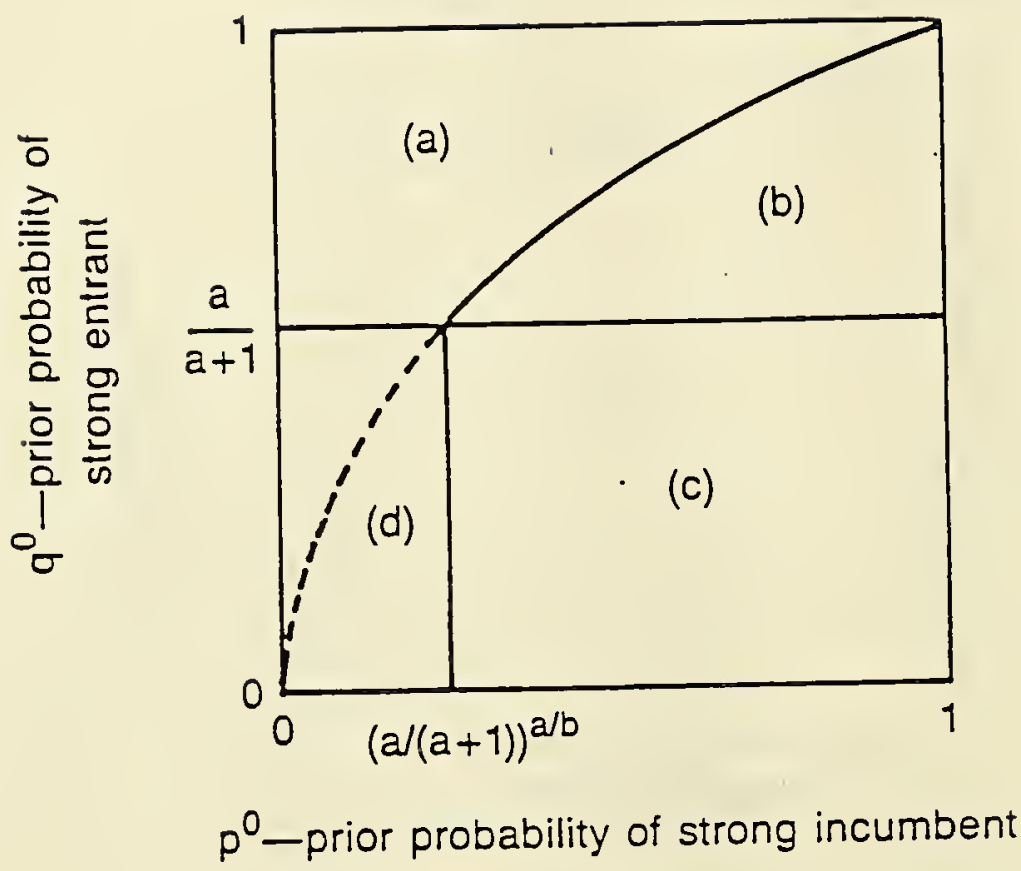

Figure 3. Isolation vs. linkage with the concession game as the single contest. In region (a), the weak incumbent is asymptotically indifferent, as it nets zero per contest in either case. In regions (b) and (c) isolation is prefered. Note that with informational linkage, the weak incumbent maintains the reputation in region (c) and gives it up in (b). In region (d), the weak incumbent prefers linkage. See Proposition 3 for further details. 


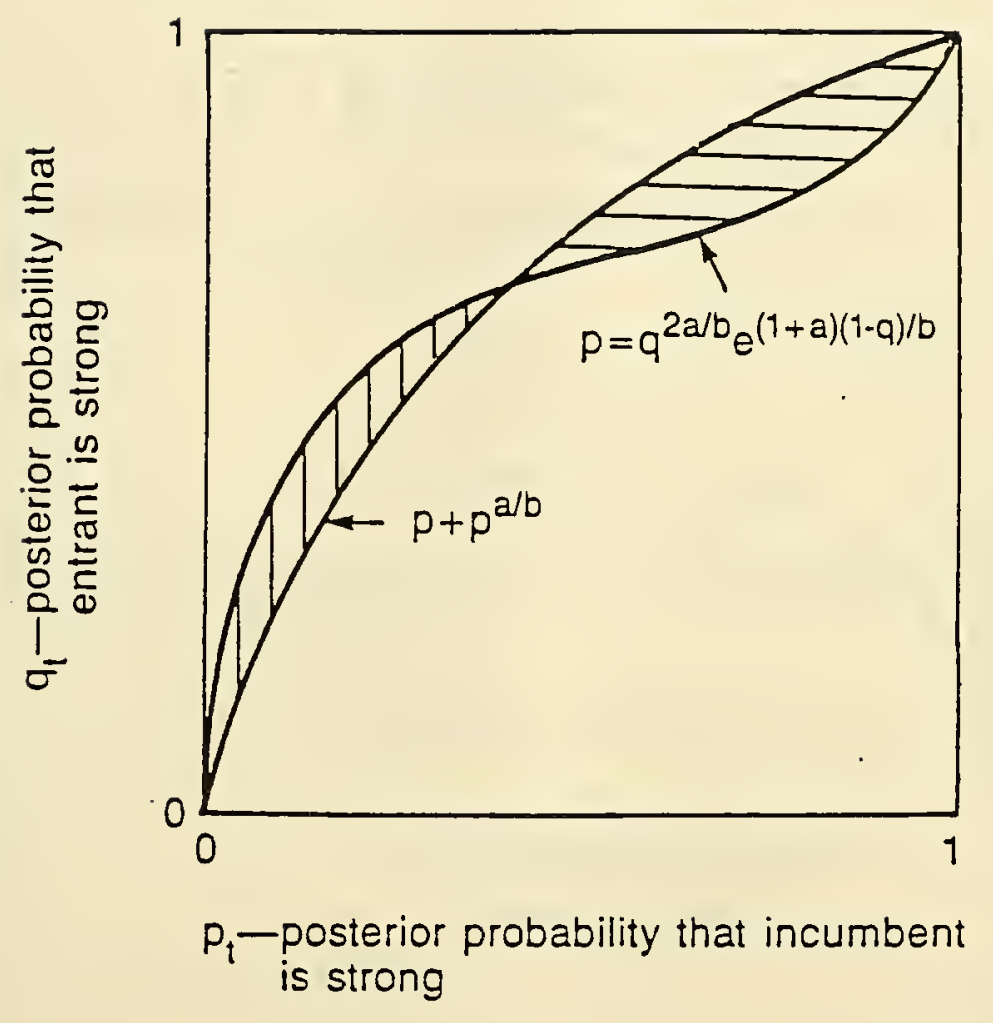

Figure 4. The equilibrium for the case $N=2$ and $a>1$. 


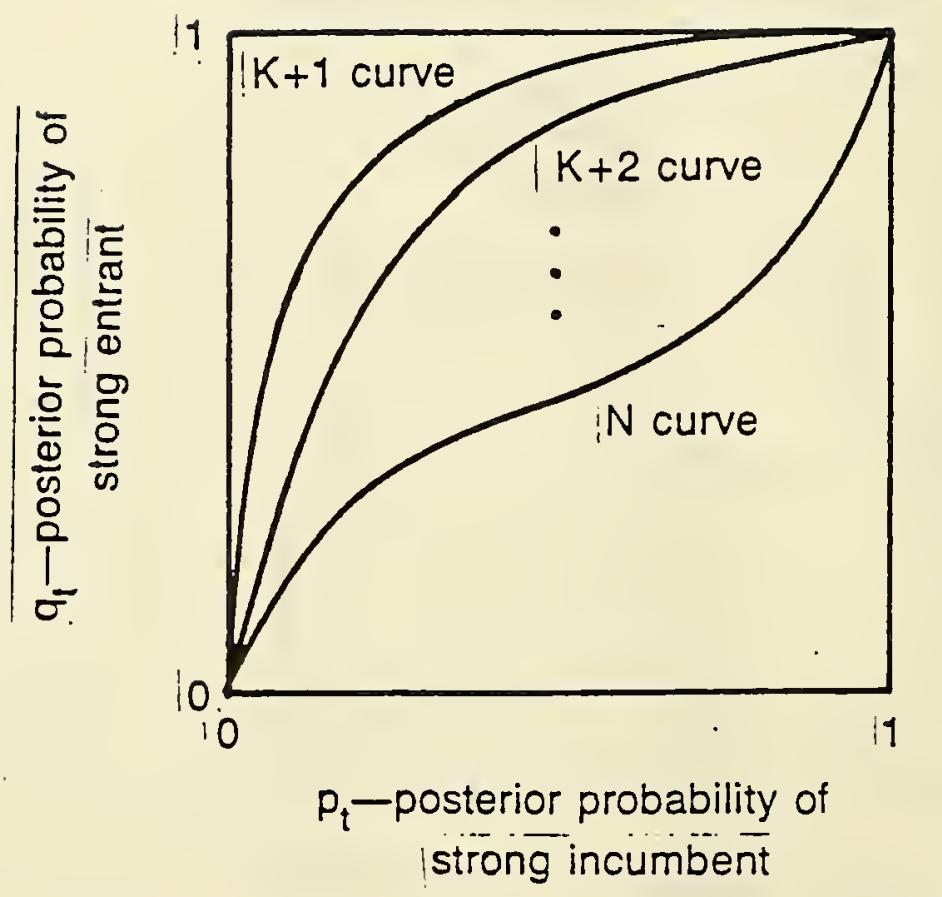

Figure 5. State space of the game with simultaneous contests and no reentry. 


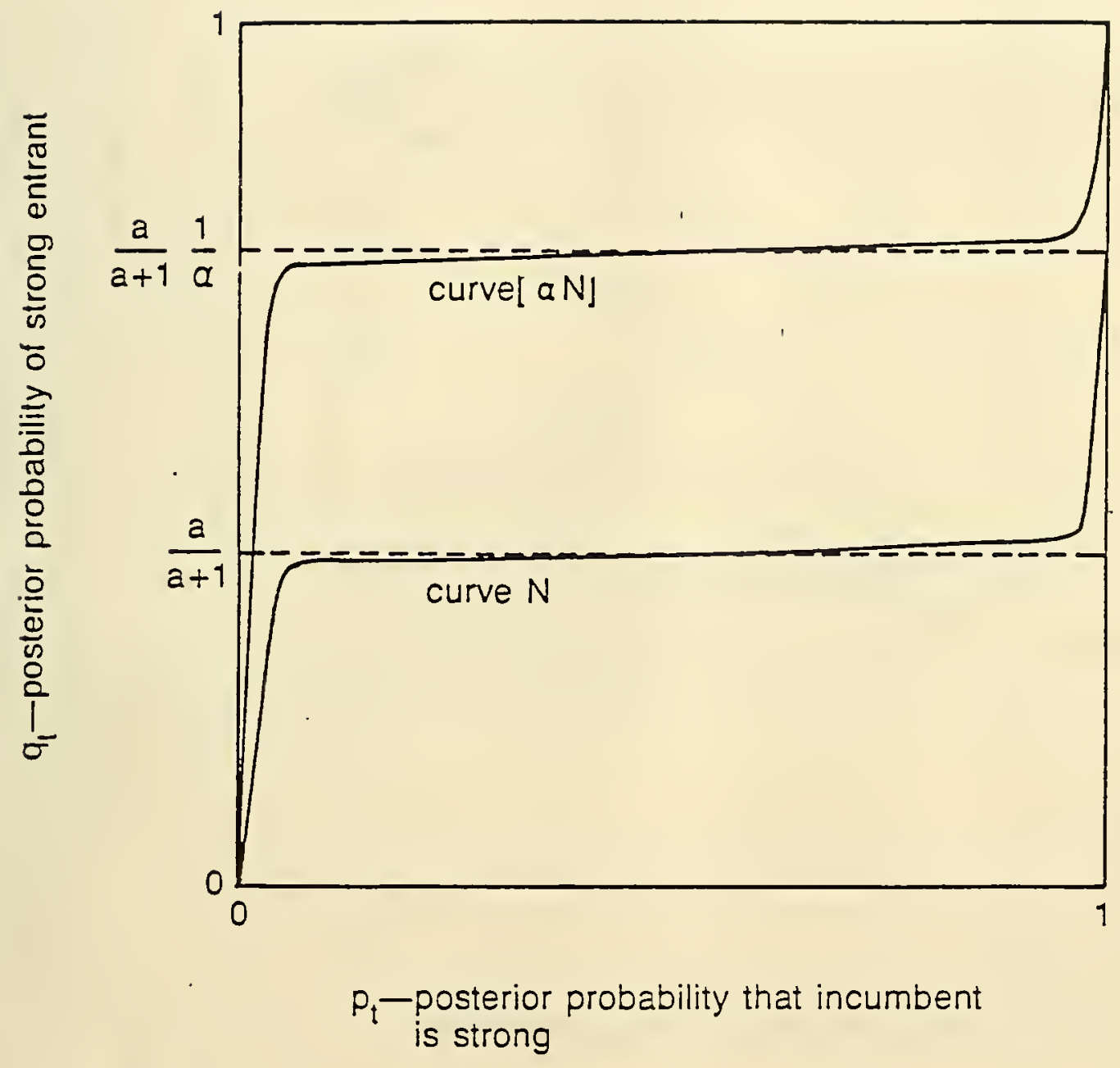

Figure 6. Position of the $\lfloor\alpha N\rfloor$ curve for large $N$ and $\alpha \in[a /(a+1), 1]$. 


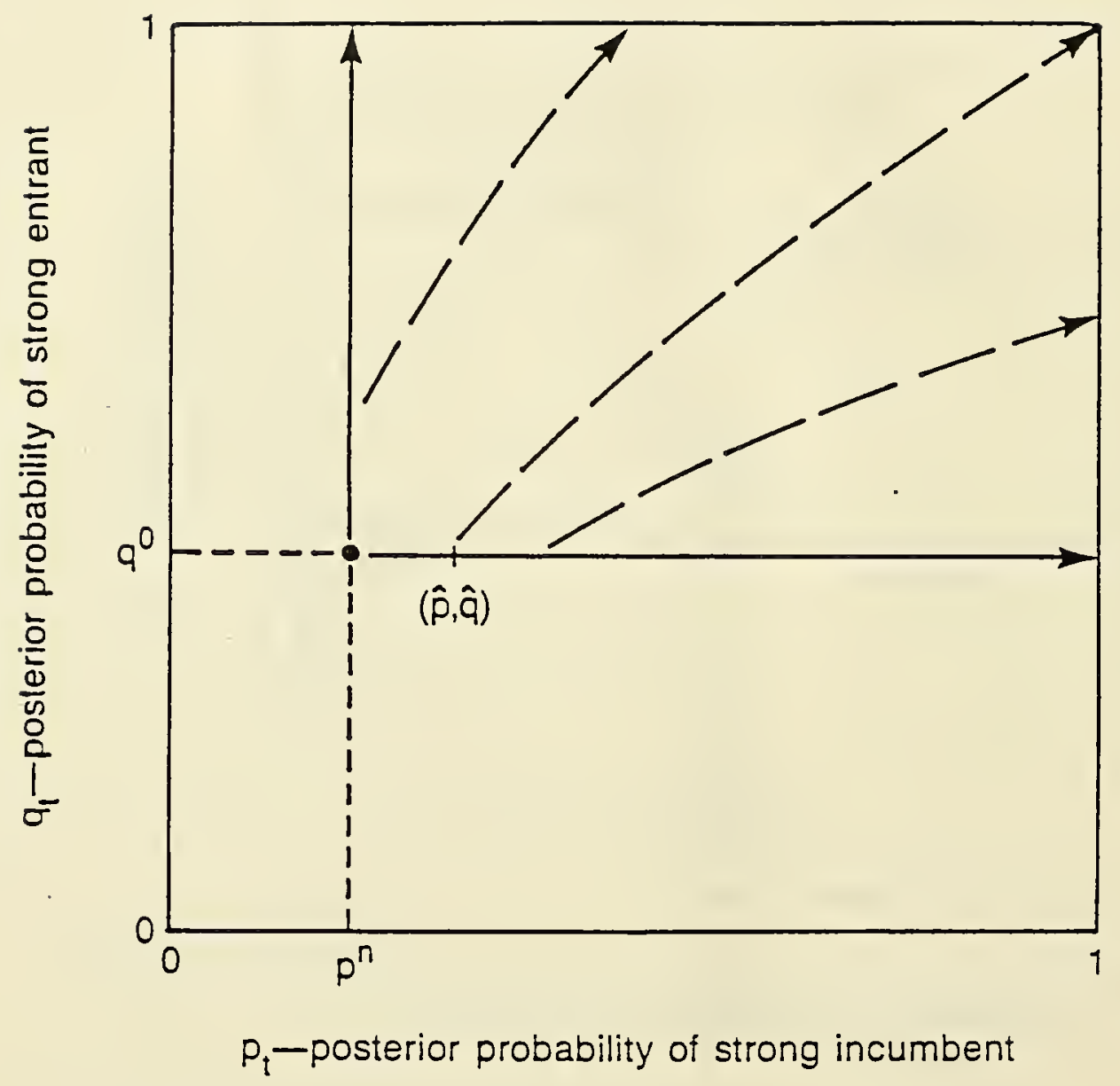

Figure A1. Solutions to the differential equations $(A .2)$ for various starting and ending points. 
Figure A2. Finding the correct terminal condition for stage $n$.

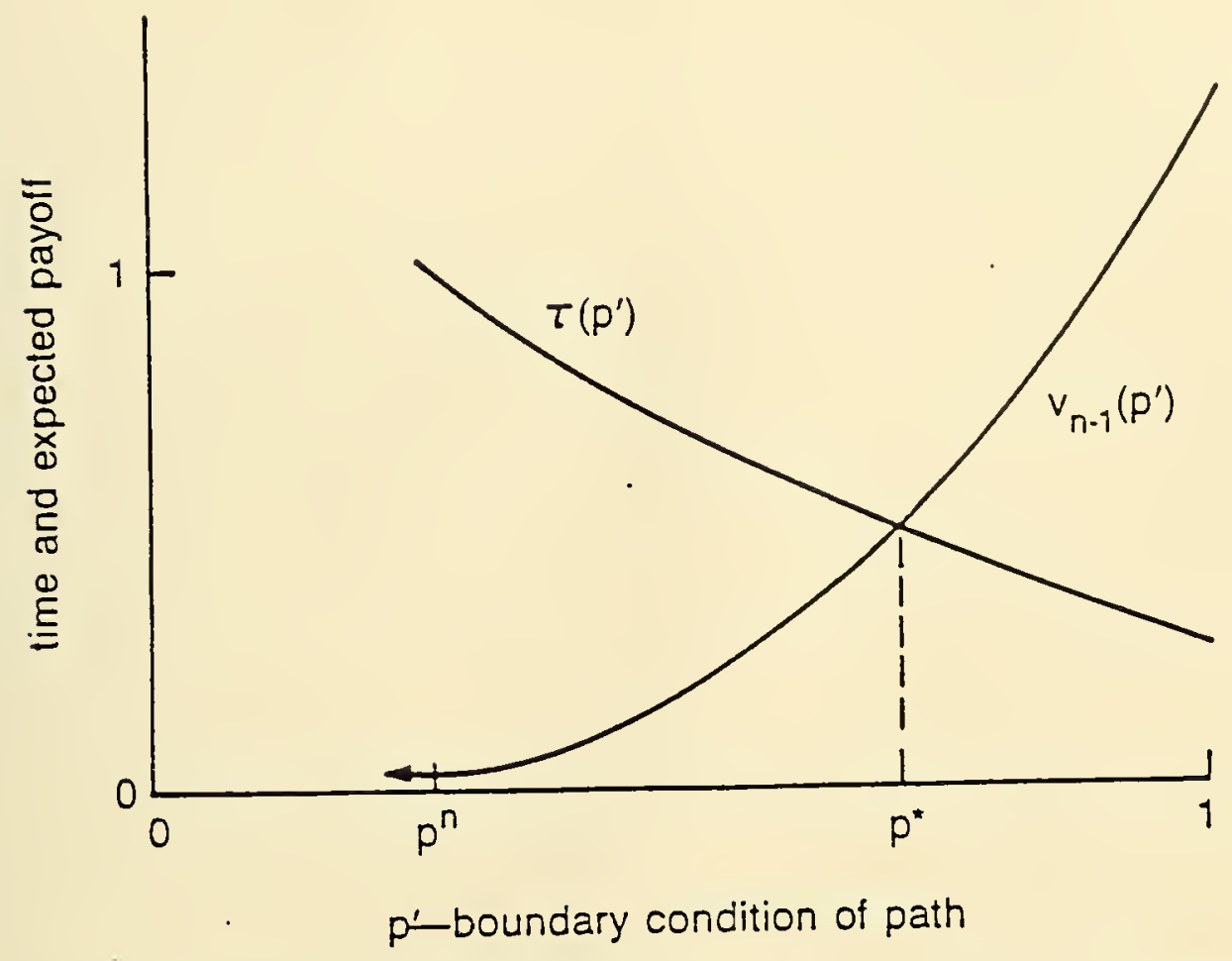

(a) In this case, the correct ending point is $\left(p^{*}, 1\right)$, where $p^{*}$ is the solution of $\tau(p)=v_{n-1}(p)$.

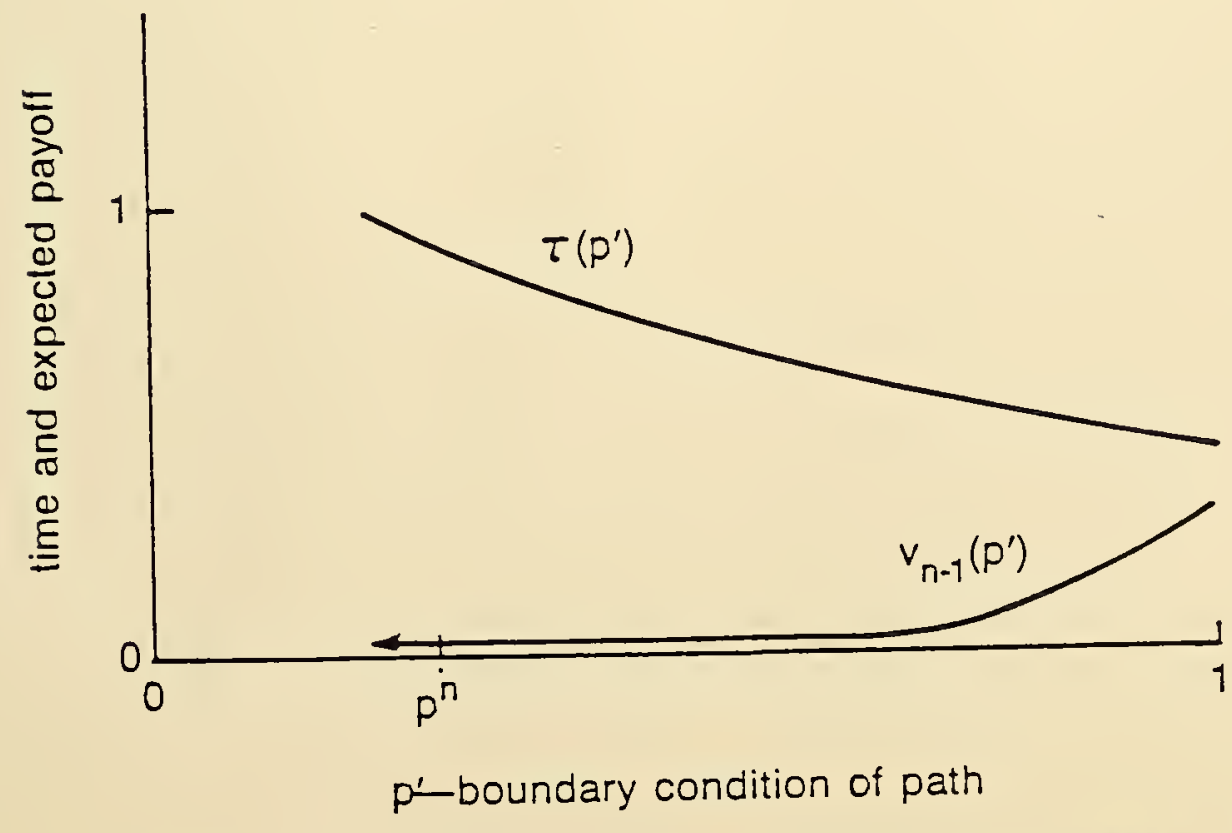

(b) In this case, where $\tau(1)>v_{n-1}(1)$, the correct ending point is $(1,1)$. 
3611095 




$3980005130 \quad 304$
4 
Hests

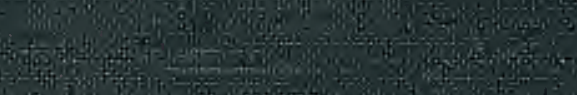

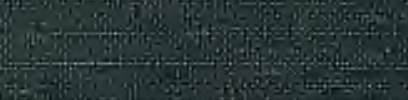

- 